\title{
THE STRENGTH OF JULLIEN'S INDECOMPOSABILITY THEOREM
}

\author{
ITAY NEEMAN
}

\begin{abstract}
Jullien's indecomposability theorem states that if a scattered countable linear order is indecomposable, then it is either indecomposable to the left, or indecomposable to the right. The theorem was shown by Montalbán to be a theorem of hyperarithmetic analysis. We identify the strength of the theorem relative to standard reverse mathematics markers. We show that it lies strictly between weak $\Sigma_{1}^{1}$ choice and $\Delta_{1}^{1}$ comprehension.
\end{abstract}

\$1. Introduction. A linear order $\left(U ;<_{U}\right)$ (denoted simply $U$ below) is scattered if it does not contain a copy of the order of rational numbers. A cut in the order $U$ is a pair $\langle L, R\rangle$ so that $L \cap R=\emptyset, L \cup R=U, L$ is closed downward (or leftward) in $<_{U}$, and $R$ is closed upward (or rightward). The cut is a decomposition of $U$ if $U$ does not embed into either one of $L, R$. The order $U$ is indecomposable if it has no decompositions, or, equivalently, if for every cut $\langle L, R\rangle$ in the order, $U$ embeds either into $L$ or into $R$.

If $U$ embeds into $L$ whenever $\langle L, R\rangle$ is a cut in $U$ with $L \neq \emptyset$, then $U$ is indecomposable to the left. Indecomposability to the right is defined similarly. Jullien [2] proved the following rather curious result:

Theorem 1.1 (Jullien [2]). Let $\left(U ;<_{U}\right)$ be a countable linear order. Suppose $\left(U ;<_{U}\right)$ is scattered and indecomposable. Then $\left(U ;<_{U}\right)$ is either indecomposable to the left, or indecomposable to the right.

Montalbán [4] initiated the search for the reverse mathematics strength of this theorem. Recall that reverse mathematics is concerned with the strength of theorems of second order number theory, also called analysis since it encompasses the first order theory of natural and real numbers. The strength of a theorem is measured in terms of the set existence axioms needed for its proof, over a base theory consisting of the basic axioms of arithmetic, some form of induction (ranging from full induction to just $\Sigma_{1}^{0}$ induction), and the first set existence schema in the list below, $\Delta_{1}^{0}$ comprehension.

There are several established axioms and axiom schemas of set existence which serve as markers of strength over this base theory. The following is a partial list of the axioms and schemas studied. When added to the base theory they result in subsystems of analysis. The list is arranged so that the resulting subsystems are in order of strictly increasing strength. The implications and, especially, non-implications needed to show this are in some cases highly non-trivial. Many

This material is based upon work supported by the National Science Foundation under Grant No. DMS-0556223 
of them can be found in Simpson [6]. Let us only comment that the fact that the strengths of the systems resulting from (5), (6), and (7) are strictly increasing is due to Steel $[7,8]$ and Van Wesep [9]. Their work is essential to the proofs in this paper.

Formulas $\varphi$ and $\psi$ in the list are allowed to have real parameters.

1. $\Delta_{1}^{0}$ comprehension, asserting for each pair of $\Sigma_{1}^{0}$ formulas $\varphi, \psi$, that if $(\forall n \in$ $\omega)(\varphi(n) \leftrightarrow \neg \psi(n))$, then the set $\{n \in \omega \mid \varphi(n)\}=\{n \in \omega \mid \neg \psi(n)\}$ exists.

2. Weak König's lemma, asserting that each infinite subtree of the binary tree has an infinite branch.

3. Arithmetic comprehension, asserting for each arithmetic $\varphi$, that the set $\{n \in \omega \mid \varphi(n)\}$ exists. Equivalently (over the base system), the Turing jump of every real exists.

4. Jump iteration, asserting that every real has a Turing jump and that iterations of the jump operator can be continued at each (countable) limit. Precisely, $(\forall x \in \mathbb{R})(\forall$ ordinal $\alpha)\left(\left(x^{(\beta)}\right.\right.$ exists for all $\left.\beta<\alpha\right) \rightarrow x^{(\alpha)}$ exists $)$.

5. Weak $\Sigma_{1}^{1}$ choice (also called arithmetic replacement), asserting for each arithmetic $\varphi$, that if $(\forall n \in \omega)(\exists$ unique $y \in \mathbb{R}) \varphi(n, y)$, then there is a sequence $\left\langle y_{n} \mid n<\omega\right\rangle$ so that $(\forall n) \varphi\left(n, y_{n}\right)$.

6. $\Delta_{1}^{1}$ comprehension, asserting for each pair of $\Sigma_{1}^{1}$ formulas $\varphi, \psi$, that if $(\forall n \in$ $\omega)(\varphi(n) \leftrightarrow \neg \psi(n))$, then the set $\{n \in \omega \mid \varphi(n)\}=\{n \in \omega \mid \neg \psi(n)\}$ exists.

7. $\Sigma_{1}^{1}$ choice, asserting for each arithmetic $\varphi$, that if $(\forall n \in \omega)(\exists y \in \mathbb{R}) \varphi(n, y)$, then there is a sequence $\left\langle y_{n} \mid n<\omega\right\rangle$ so that $(\forall n) \varphi\left(n, y_{n}\right)$.

8. Arithmetic transfinite recursion, asserting for each arithmetic formula, that definition by comprehension using the formula can be iterated transfinitely along any (countable) wellorder.

9. $\Pi_{1}^{1}$ comprehension, asserting for each $\Pi_{1}^{1}$ formula $\varphi$, that $\{n \in \omega \mid \varphi(n)\}$ exists.

The systems resulting from (1), (2), (3), (8), and (9) are the big five systems of reverse mathematics. Over the years they have been shown to capture the strength of many theorems of analysis, see [6].

The systems resulting from (4), (5), (6), and (7) are all systems of hyperarithmetic analysis. $T$ is a theory, or system, of hyperarithmetic analysis, if on the one hand it is strong enough that every $\omega$-model of $T$ is closed under joins and hyperarithmetic reducibility, and on the other hand it is weak enough that it holds in $\operatorname{HYP}(x)$ for every real $x$. There are systems of hyperarithmetic analysis that have been studied and do not appear in the list above, see Montalbán [4] and [3] for details. But none of them lies strictly between (5) and (6)

Montalbán [4] proved that Jullien's indecomposability theorem, which he termed INDEC, is a theorem of hyperarithmetic analysis. More precisely he showed that it follows from $\Delta_{1}^{1}$ comprehension, and its $\omega$-models are closed under joins and under the $\alpha$ th Turing jump operator for each ordinal $\alpha$ that belongs to the model. The result is significant because it makes INDEC the first "natural" mathematical theorem shown to be a theorem of hyperarithmetic analysis. Natural here is taken to mean that the theorem had been published independently of reverse mathematics. INDEC had been published in [1] and [5]. 
Montalbán's work left the precise strength of INDEC open. It remained open whether INDEC implies jump iteration (as Montalbán's proof mentioned above applied only to $\omega$ models), whehter it was comparable with weak $\Sigma_{1}^{1}$ choice at all, and whether it was equivalent to $\Delta_{1}^{1}$ comprehension. We address the strength of INDEC in this paper. We show that:

TheOREM 1.2. (Over $\mathrm{RCA}_{*}$, see below.) INDEC implies weak $\Sigma_{1}^{1}$ choice.

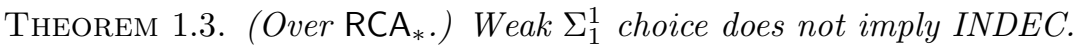

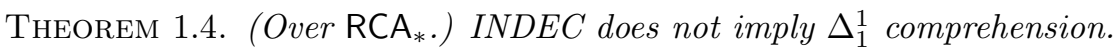

Implications in all theorems are in the base theory $\mathrm{RCA}_{*}$, consisting of the basic axioms of arithmetic, $\Delta_{1}^{0}$ comprehension, and $\Sigma_{1}^{1}$ induction. The models witnessing Theorems 1.3 and 1.4 are $\omega$-models, and so the theorems continue to hold with base theories that allow stronger induction.

Since INDEC follows from $\Delta_{1}^{1}$ comprehension, the theorems place INDEC strictly between weak $\Sigma_{1}^{1}$ choice and $\Delta_{1}^{1}$ comprehension. There are no established set existence schemas between these two markers, so this is as precise an analysis of the strength of INDEC as one could hope for.

Theorem 1.2 is proved in Section 2. The argument there is simply a proof in the base theory. Theorems 1.3 and 1.4 are proved in Sections 3 and 4. These theorems require constructions of models satisfying weak $\Sigma_{1}^{1}$ choice plus the negation of INDEC, and INDEC plus the negation of $\Delta_{1}^{1}$ comprehension, respectively. The models are constructed through a use of Steel's forcing, developed in $[7,8]$. We shall say more on this when we get to Section 3 .

§2. INDEC implies weak $\Sigma_{1}^{1}$ choice. We prove that INDEC implies weak $\Sigma_{1}^{1}$ choice in the system $\mathrm{RCA}_{*}$, consisting of the basic axioms of arithmetic, $\Sigma_{1}^{1}$ induction, and $\Delta_{1}^{0}$ comprehension. Except for the very first claim, the proof can be carried out in the weaker $\mathrm{RCA}_{0}$, in which induction is restricted to $\Sigma_{1}^{0}$ formulas.

During the proof we talk about elements of $\omega^{\omega}$ (these are the reals, following standard abuse of notation), sequences, both finite and of length $\omega$, with elements from $\omega \cup \mathbb{R}$, and trees (sets of sequences closed under initial segments) on countable sets. All these objects can be coded by subsets of $\omega$, so that the axioms of $\mathrm{RCA}_{*}$ apply to them through the coding. We work with the objects themselves, rather than the codes, to simplify notation.

Weak $\Sigma_{1}^{1}$ choice states that if $\varphi$ is an arithmetic formula such that $(\forall n \in$ $\omega)(\exists ! y \in \mathbb{R}) \varphi(n, y)$, then there exists a sequence $\left\langle y_{n} \mid n<\omega\right\rangle$ so that $(\forall n) \varphi\left(n, y_{n}\right)$ By standard arguments this statement is equivalent to its restriction to $\Pi_{1}^{0}$ formulas. We prove this restriction.

Fix then a $\Pi_{1}^{0}$ formula $\varphi$ so that $(\forall n)(\exists ! y) \varphi(n, y)$. Say $\varphi=(\forall i) \psi(n, i, x)$ where $\psi$ has only bounded quantifiers. Let $S_{n}$ be the tree consisting of tuples $s \in \omega^{<\omega}$ so that $\psi(n, i, s)$ holds for all $i<\operatorname{lh}(s)$. That the sequence of trees $\left\langle S_{n} \mid n<\omega\right\rangle$ exists follows from $\Delta_{1}^{0}$ comprehension. Each of the trees $S_{n}$ has a unique infinite branch, namely the unique real $y$ so that $\varphi(n, y)$.

Claim 2.1. For all $k<\omega$, there exists a unique sequence $\left\langle y_{0}, \ldots, y_{k-1}\right\rangle$ so that $(\forall n<k) \varphi\left(n, y_{n}\right)$. 
ProOF. Uniqueness is clear from the uniqueness of $y$ such that $\varphi(n, y)$ for each $n$. Existence is easily proved by induction on $k$, using the fact that $(\forall n)(\exists y) \varphi(n, y)$.

Note that we are using $\Sigma_{1}^{1}$ induction in the proof of Claim 2.1. This is our only use of $\Sigma_{1}^{1}$ induction. The rest of the proof that INDEC implies weak $\Sigma_{1}^{1}$ choice is entirely in $\mathrm{RCA}_{0}$.

We shall construct from $\left\langle S_{n} \mid n<\omega\right\rangle$ a scattered linear order $\left(U ;<_{U}\right)$ which we shall prove embeds into both a non-trivial left tail-end and a non-trivial right tail-end of itself. By INDEC it will follow that the linear order is decomposable, and from the cut witnessing this we shall construct a sequence of infinite branches through the trees $S_{n}$.

Let $I$ be the integers equipped with the following linear order $<_{I}$ : $-n<_{I}$ $-m<_{I} 0<_{I} m<_{I} n$ for all $0<n<m<\omega$. The order thus has a middle point, 0 , with a part of order type $\omega$ to its left, and a part of order type $\omega^{*}$ to its right. It is displayed in Diagram 1.

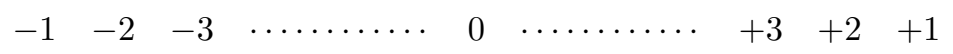

Diagram 1. The order $<_{I}$

By $I^{<\omega}$ we mean the tree of finite sequences of elements of $I$. The BrouwerKleene order on $I^{<\omega}$ is defined using the order $<_{I}$. Precisely, it is the linear order $<_{L}$ determined by the conditions $q \frown\langle i\rangle<_{L} q$, and $q \frown\langle i\rangle<_{L} q \frown\langle j\rangle$ iff $i<_{I} j$. Note that we are using the order $<_{I}$, not the ordinary order on $\mathbb{Z}$, and we talk about $I^{<\omega}$ rather than $\mathbb{Z}^{<\omega}$ to emphasize this.

We regard each subset $A$ of $I^{<\omega}$ as a linear order. precisely it is the linear order $\left(A ;<_{L} \uparrow A\right)$, but we usually suppress mention of $<_{L}$. Thus when we write, for example, that $A$ embeds into $B$ we mean that $\left(A ;<_{L}\lceil A)\right.$ embeds into $\left(B ;<_{L}\lceil B)\right.$, and when we write that $\mathbb{Q}$ embeds into $A$ we mean that it embeds into $\left(A ;<_{L}\lceil A)\right.$.

For each node $p \in I^{<\omega}$ let $\operatorname{nbd}(p)$ consist of all nodes which extend $p$ strictly. Let $\operatorname{Lnbd}(p)$ consist of all nodes to the left $\operatorname{nbd}(p)$. Precisely, $q \in \operatorname{Lnbd}(p)$ if $q<_{L} r$ for each $r \in \operatorname{nbd}(p)$, or equivalently, $q<_{L} p$ and $q \notin \operatorname{nbd}(p)$. Define $\operatorname{Rnbd}(p)$ similarly: $q \in \operatorname{Rnbd}(p)$ if $q>_{L} r$ for each $r \in \operatorname{nbd}(p)$, or equivalently, $q \geq_{L} p$.

$\operatorname{Rnbd}(p)$ is a tree. $\operatorname{Lnbd}(p)$ is not a tree, but only because it is missing the strict initial segments of $p$. Let $\operatorname{sInSeg}(p)=\{p\lceil i \mid i<\operatorname{lh}(p)\}$. Then $\operatorname{Lnbd}(p) \cup \operatorname{sinSeg}(p)$ is a tree.

For a set $C \subseteq \omega$ and a sequence $t$ of length $\leq \omega$, define $t\lceil C$ to be the sequence $\left\langle t\left(n_{i}\right) \mid i<l\right\rangle$ where $\left\langle n_{i} \mid i<l\right\rangle$ is an increasing enumeration of $C \cap \operatorname{lh}(t)$.

Let Even and $O d d$ be the sets of even and odd numbers respectively. Define $t_{\text {Even }}$ to be $t\left\lceil\right.$ Even, and define $t_{\text {Odd }}$ similarly.

Let $\left\langle C_{i} \mid i<\omega\right\rangle$ be a recursive partition of $\omega$ into infinitely many infinite sets, with $C_{0}=$ Even and with the property that $n \subseteq \bigcup_{i<n} C_{i}$ for each $n$. By the product $\Pi_{i<\omega} T_{i}$ of trees $T_{i}, i<\omega$, we mean the tree $T$ consisting of sequences $t$ so that $t\left\lceil C_{i}\right.$ is a node in $T_{i}$ for each $i<\omega$. Given branches $x_{i}$ through $T_{i}$, define $\Pi_{i<\omega} x_{i}$ to be the branch $x$ through $T$ determined by the condition 
$x\left\lceil C_{i}=x_{i}\right.$. For $n<\omega$ we define the products $\Pi_{i<n} T_{i}$ and $\Pi_{i<n} x_{i}$ similarly, using the partition $C_{0}, \ldots, C_{n-2}, \bigcup_{i \geq n-1} C_{i}$. We adopt these definitions for products since they make it easy to relate the Brouwer-Kleene order on the trees $T_{i}$ to the Brouwer-Kleene order on products $T$. More precisely, if $T_{i} \subseteq I^{<\omega}$, then $T \subseteq I^{<\omega}$ too. The definitions are such that a product $T_{0} \times T_{1}=\Pi_{i<2} T_{i}$ consists precisely of nodes $t$ so that $t_{\text {Even }} \in T_{0}$ and $t_{O d d} \in T_{1}$.

Let $S^{*}$ denote the product $\Pi_{i<\omega} S_{i}$. Let $S_{n}^{*}$ denote the product $\Pi_{i<n} S_{i}$. The fact that $n \subseteq \bigcup_{i<n} C_{i}$ implies that $S^{*}$ and $S_{n}^{*}$ agree on nodes of lengths $\leq n$.

For a node $t$ in a tree $T$ let $T(t)=\left\{r \mid t^{\frown} r \in T\right\}$. Let $\mathcal{F}$ be the function on $S^{*}$ defined by $\mathcal{F}(s)=S_{k}^{*}(s)$ where $k=\operatorname{lh}(s)$ (whence $s \in S_{k}^{*}$ ). The existence of $S^{*}$ and $\mathcal{F}$ follows from the existence of the sequence $\left\langle S_{i} \mid i<\omega\right\rangle$, using $\Delta_{1}^{0}$ comprehension. $S^{*}$ and $\mathcal{F}$ have the properties given by the following claim, and these are their only properties that we shall use, to show that INDEC implies the existence of a branch through $S^{*}$.

Claim 2.2. $S^{*}$ is a tree on $\omega$, and for each $s \in S^{*}, \mathcal{F}(s)$ is a tree on $\omega$. The trees are such that:

1. If $s \in S^{*}$ can be extended to a branch through $S^{*}$, then there is a branch through $\mathcal{F}(s)$.

2. If $s, s^{\prime} \in S^{*}$ are incompatible, then it cannot be that both $\mathcal{F}(s)$ and $\mathcal{F}\left(s^{\prime}\right)$ have branches.

3. For every $k$ there is $s \in S^{*}$ of length $k$ so that $\mathcal{F}(s)$ has a branch. (This $s$ is unique by the previous condition.)

Proof. The existence claimed in condition (3) follows from the definitions using the existence in Claim 2.1. If $\left\langle y_{0}, \ldots, y_{k-1}\right\rangle$ is such that $(\forall n<k) \varphi\left(n, y_{n}\right)$, then $y_{k}^{*}=\Pi_{i<k} y_{i}$ is a branch through $S_{k}^{*}$, and $s=y_{k}^{*}\lceil k$ witnesses condition (3).

Suppose $s, s^{\prime} \in S^{*}$ are incompatible. Let $j<\min \left(\operatorname{lh}(s), \operatorname{lh}\left(s^{\prime}\right)\right)$ be such that $s(j) \neq s^{\prime}(j)$, and let $i<\min \left(\operatorname{lh}(s), \operatorname{lh}\left(s^{\prime}\right)\right)$ be such that $j \in C_{i}$. Suppose $\mathcal{F}(s)$ and $\mathcal{F}\left(s^{\prime}\right)$ both have branches. Then $s$ can be extended to a branch through $S_{\mathrm{lh}(s)}^{*}$, which must have the form $\Pi_{n<\operatorname{lh}(s)} y_{n}$ with $y_{n} \in S_{n}$, and $y_{n}$ extending $s\left\lceil C_{n}\right.$ for each $n<\operatorname{lh}(s)$. Similarly there is a branch through $S_{\mathrm{lh}\left(s^{\prime}\right)}^{*}$, of the form $\Pi_{n<\operatorname{lh}\left(s^{\prime}\right)} y_{n}^{\prime}$, with $y_{n}^{\prime} \in S_{n}$ and $y_{n}^{\prime}$ extending $s^{\prime} \uparrow C_{n}$ for each $n<\operatorname{lh}\left(s^{\prime}\right)$. Both $y_{i}$ and $y_{i}^{\prime}$ are branches through $S_{i}$, and since the tree has a unique branch it follows that $y_{i}=y_{i}^{\prime}$. But from the fact that $s$ and $s^{\prime}$ disagree on $j \in C_{i}$ it follows that $y_{i} \neq y_{i}^{\prime}$. This contradiction completes the proof of condition (2).

For condition (1), suppose $s$ can be extended to a branch $y^{*}$ through $S^{*}$. Then $y^{*}$ has the form $\Pi_{n<\omega} y_{n}$ with $y_{n}$ a branch through $S_{n}$. Let $y_{k}^{*}=\Pi_{n<k} y_{n}$. Then $y_{k}^{*}$ is a branch through $S_{k}^{*}$, and agrees with $y^{*}$ to $k$. Taking $k=\operatorname{lh}(s)$ it follows that $y_{k}^{*} \uparrow[k, \omega)$ is a branch through $\mathcal{F}(s)$.

Our goal is to prove the existence of a branch through $S^{*}$. For motivational purposes it is convenient to talk about the unique branch through $S^{*}$, even before we prove its existence. Let $y^{*}$ denote this branch. (We emphasize that we are talking about it here only for motivational purposes, as we have not yet proved it exists.) We intend to construct a tree $U \subseteq I^{<\omega}$ with the following properties:

1. $U$ has only one infinite branch $b$. 
2. If $q$ is a node of $U$ to the right of $b$, then $U$ can be embedded into the nodes of $U$ to the left of $q$. Similarly if $q$ is to the left of $b$, then $U$ can be embedded into the nodes of $U$ to the right of $q$.

3. $b$ codes $y^{*}$.

It follows from property (1) that $U$ is scattered (meaning that $\left(U ;<_{L} \mid U\right)$ is scattered). From property (2) using INDEC it follows that $U$ must be decomposable into two parts, the part to the left of $b$ and the part to the right of $b$, and in particular it follows that $b$ exists. Using property (3) it follows finally that from this decomposition one can construct the branch $y^{*}$ through $S^{*}$.

The definition of $U$, which we give next, is recursive. We shall comment on the recursion below. It is clear from the definition that the existence of $U$ follows from the existence of $S^{*}$ and $\mathcal{F}$ using $\Sigma_{1}^{0}$ induction (to show that the recursive recipe for identifying nodes is $\Delta_{1}^{0}$ ) and $\Delta_{1}^{0}$ comprehension.

DEFinition 2.3. $U$ has four types of nodes, primary nodes, middle children of primary nodes, left descendants of primary nodes, and right descendants of primary nodes.

1. If $s=\left\langle a_{0}, \ldots, a_{k-1}\right\rangle$ is a node in $S^{*}$ then $p=\left\langle 0, a_{0}, 0, a_{1}, \ldots, 0, a_{k-1}\right\rangle$ is a primary node of $U$. We refer to it as the primary node labelled with $s$, and denote it $p(s)$.

2. $p(s)^{\frown}\langle 0\rangle$ is the middle child of $p(s)$. (The children of $p(s) \frown\langle 0\rangle$ in turn are the primary nodes $p(s) \frown\langle 0, e\rangle$ for $e$ so that $s \frown\langle e\rangle \in S^{*}$.)

3. The primary node $p(s)=\left\langle 0, a_{0}, 0, a_{1}, \ldots, 0, a_{k-1}\right\rangle$ has additional children of two kinds, $p(s)^{\frown}\langle-n\rangle$ and $p(s) \frown\langle+n\rangle$, for $0<n<\omega$. We call these the left and right children of $p(s)$ respectively.

4. Below a left child $p(s) \frown\langle-n\rangle$, with $\ln (s)=k$, there sits the tree $\mathcal{F}(s) \times$ $\left(U \cap T_{\text {Left }}\right)$, where $T_{\text {Left }}$ is the tree $\operatorname{Lnbd}(p(s)) \cup \operatorname{sinSeg}(p(s))$. Precisely, the left descendants of $p(s)$ are nodes of the form $p(s)^{\frown}\langle\langle-n\rangle-t$ where:

(a) $t_{\text {Even }} \in \mathcal{F}(s)$.

(b) $t_{\text {Odd }} \in U$ and $t_{\text {Odd }} \in \operatorname{Lnbd}(p(s)) \cup \operatorname{sInSeg}(p(s))$.

(The strict initial segments of $p(s)$ are added to make $T_{\text {Left }}$ a tree.)

5. Similarly, below a right child $p(s)^{\mathcal{C}}\langle+n\rangle$ there sits the tree $\mathcal{F}(s) \times(U \cap$ $T_{\text {Right }}$ ), where $T_{\text {Right }}=\operatorname{Rnbd}(p(s))$. (There is no need to add the strict initial segments of $p(s)$ on this side, as they already belong to $\operatorname{Rnbd}(p(s))$.)

This completes the definition of $U$.

To determine which nodes of length $l$ belong to $U$, we assume knowledge of the restriction of $U$ to shorter nodes. This knowledge is needed in the definition of descendants of the left and right children of $p(s)$, namely in conditions (4) and (5) of the definition. To determine whether $p(s)^{\frown}\langle \pm n\rangle \frown t(n>0)$ belongs to $U$ we must determine whether $t_{\text {Odd }}$ belongs to $U$, and we can do this since $t_{\text {Odd }}$ is shorter than $p(s) \frown\langle \pm n\rangle \frown t$.

Claim 2.4. Let $s \in S^{*}$ be such that there exists a branch through $\mathcal{F}(s)$. Let $p=$ $p(s)$, and let $n>0$. Then $U$ embeds into its left tail-end $\left\{q \in U \mid q<_{L} p \curlyvee\langle+n\rangle\right\}$, and into its right tail-end $\left\{q \in U \mid q>_{L} p \frown\langle-n\rangle\right\}$.

Proof. We define an embedding $\pi$ of $U$ into $\left\{q \in U \mid q>_{L} p-\langle-n\rangle\right\}$. An embedding of $U$ into $\left\{q \in U \mid q<_{L} p^{\frown}\langle+n\rangle\right\}$ can be obtained similarly. 
We divide $U$ into three components, corresponding to the following three parts of $I^{<\omega}:(1) K_{1}=\operatorname{Lnbd}(p)$; (2) $K_{2}=\bigcup_{m>0} \operatorname{nbd}(p \frown\langle-m\rangle)$; and (3) the rest, meaning $K_{3}=\operatorname{nbd}\left(p^{\frown}\langle 0\rangle\right) \cup \bigcup_{m>0} \operatorname{nbd}\left(p^{\frown}\langle+m\rangle\right) \cup \operatorname{Rnbd}(p)$. We define $\pi$ separately on each component.

Let $z$ be a branch through $\mathcal{F}(s)$.

First, for $t \in U \cap K_{1}$ define $\pi(t)=p^{-}\langle-(n+1)\rangle \frown((z \nmid \ln (t)) \times t) . \pi(t)$ belongs to $U$ by condition (4) in Definition 2.3 .

Next, for $t=p \frown\langle-m\rangle \frown r \in U \cap K_{2}(m>0)$, define $\left.\pi(t)=p \frown\langle-(m+n+1)\rangle\right\urcorner r$.

Finally, for $t \in U \cap K_{3}$ define $\pi(t)=t$.

Thus $\pi$ embeds $U \cap \operatorname{Lnbd}(p)$ into $\operatorname{nbd}\left(p^{\frown}\langle-(n+1)\rangle\right)$, shifts $U \cap \operatorname{nbd}\left(p^{\frown}\langle-m\rangle\right)$ to $\operatorname{nbd}\left(p^{\frown}\langle-(m+n+1)\rangle\right)$, and fixes all nodes from $\operatorname{nbd}\left(p^{\frown}\langle 0\rangle\right)$ rightwards. $\dashv$

Claim 2.4 leads to the motivational property (2) above, with $b=\overrightarrow{0} \times y^{*} . \overrightarrow{0}$ here is the sequence $\langle 0,0, \ldots\rangle$, and it is clear from the definition of $U$ that $\overrightarrow{0} \times y^{*}$ is a branch through the tree. (Of course we cannot say this yet, except for motivational purposes, as we have not shown that $y^{*}$ exists.) The motivational property (3) is clear. The claims below establish the motivational property (1), but we prove them, as we must, without reference to $y^{*}$ and $b$.

The key to the proof that $U$ has at most one branch is the fact that nodes in $U$ code nodes in the trees $S^{*}$ and $\mathcal{F}(s)$ for $s \in S^{*}$. If $x$ is a branch through $U$, consisting only of primary nodes and their middle children, then $x_{\text {Odd }}$ is a branch through $S^{*}$. If $x$ includes a left or right child $x\left\lceil 2 k^{\frown}\langle \pm n\rangle\right.$ of a primary node, then $x_{\text {Odd }}\left\lceil[k+1, \omega)\right.$ is a branch through $\mathcal{F}\left(x_{\text {Odd }}\lceil k)\right.$. Moreover, in this case $x_{E v e n}$ has the form $\langle 0, \ldots, 0, \pm n\rangle \frown z$, where $z$ itself is a branch through $U$, and therefore $z$ codes more branches, through $S^{*}$ and/or $\mathcal{F}(s)$ for $s \in S^{*}$. Using this we shall ultimately derive uniqueness for branches of $U$ from the uniqueness given by Claim 2.2.

First, we fix some tools for obtaining all these branches through trees $S^{*}$ and $\mathcal{F}(s)$ from branches through $U$.

Define partial functions $G$ and $H$, acting on pairs $\langle q, i\rangle$ with $q \in U$ and $i \in \omega$, as follows:

1. If $q=p(s)$ is a primary node and $i=0$, then $G(q, i)$ and $G(q \frown\langle 0\rangle, i)$ are both equal to $s$, and $H(q, i)$ and $H(q \frown\langle 0\rangle, i)$ are both equal to be the symbol $*$. For all other values of $i$ the functions are undefined.

2. If $q=p(s) \frown\langle \pm n\rangle \frown r(n>0)$ then:

(a) $G(q, 0)=s$, and $H(q, 0)=r_{\text {Even }}$.

(b) $G(q, i+1)$ and $H(q, i+1)$ are equal to $G\left(r_{O d d}, i\right)$ and $H\left(r_{O d d}, i\right)$ respectively.

Claim 2.5. The functions $G$ and $H$ have the following properties:

1. $G$ and $H$ are defined on the same domain.

2. $G(q, i)$, if defined, is a node in $S^{*}$. If $H(q, i)$ is defined and not equal to *, then it is a node in $\mathcal{F}(G(q, i))$.

3. If $q$ and $q^{\prime}$ are incompatible primary nodes, then $G(q, 0)$ and $G\left(q^{\prime}, 0\right)$ are defined and are incompatible nodes of $S^{*}$.

4. If $G(q, i)$ and $H(q, i)$ are defined and $q^{\prime}$ extends $q$, then $G\left(q^{\prime}, i\right)$ and $H\left(q^{\prime}, i\right)$ are both defined, $G\left(q^{\prime}, i\right)$ extends $G(q, i)$, and $H\left(q^{\prime}, i\right)$ extends $H(q, i)$ if the latter is not $*$. If in addition $\operatorname{lh}\left(q^{\prime}\right) \geq \operatorname{lh}(q)+2^{i+1}$, then: 
(a) If $H\left(q^{\prime}, i\right)=*$ then $G\left(q^{\prime}, i\right)$ extends $G(q, i)$ strictly.

(b) If $H(q, i) \neq *$ then $G\left(q^{\prime}, i\right)=G(q, i), H\left(q^{\prime}, i\right) \neq *$, and $H\left(q^{\prime}, i\right)$ extends $H(q, i)$ strictly.

Proof. By induction on $i$, using the definitions of $G, H$, and $U$.

Claim 2.6. Let $x$ and $x^{\prime}$ be two branches through $U$. Then either $x=x^{\prime}$, or else there is $d, d^{\prime}, i, i^{\prime} \in \omega$ so that $G\left(x\lceil d, i)\right.$ and $G\left(x^{\prime}\left\lceil d^{\prime}, i^{\prime}\right)\right.$ are both defined and are incompatible.

Proof. We prove by induction on $e$ that either $x\left\lceil 2 e=x^{\prime} \uparrow 2 e\right.$, or else there exist $d, d^{\prime}, i, i^{\prime}$ as in the claim. Note that this is a $\Sigma_{1}^{0}$ statement, so we are free to use induction.

The statement is clear for $e=0$, as $x\left\lceil 0=x^{\prime}\lceil 0\right.$.

Suppose the statement is known for $e$. Suppose $x\left\lceil 2 e+2 \neq x^{\prime} \uparrow 2 e+2\right.$. We prove the existence of $d, d^{\prime}, i, i^{\prime}$.

If $x\left\lceil 2 e+2\right.$ and $x^{\prime}\left\lceil 2 e+2\right.$ are both primary, then $G\left(x\lceil 2 e+2,0)\right.$ and $G\left(x^{\prime}\lceil 2 e+2,0)\right.$ are defined and incompatible by condition (3) of Claim 2.5. Letting $d=d^{\prime}=$ $2 e+2$ and $i=i^{\prime}=0$ proves the claim.

Suppose then one of the nodes, say $x\lceil 2 e+2$ for definitiveness, is not primary. Let $k \leq e$ be such that $x\lceil 2 k$ is primary, and $x(2 k) \neq 0$. For definitiveness suppose that $x(2 k)=-n<0$, so that $x\lceil 2 k+1$ is a left child of $x\lceil 2 k$. Let $p=x\lceil 2 k$, and let $s$ be such that $p=p(s)$. Then $x$ has the form $\left.p^{\frown}\langle-n\rangle\right\urcorner h$, where $h_{\text {Even }}$ is a branch of $\mathcal{F}(s)$, and $h_{\text {Odd }}$ is a branch of $U \cap(\operatorname{Lnbd}(p) \cup \operatorname{sinSeg}(p))$.

If $x^{\prime}$ does not extend $p$, then $x \nmid 2 k \neq x^{\prime}\lceil 2 k$, and since $k \leq e$ our induction hypothesis applies, producing the required $d, d^{\prime}, i, i^{\prime}$. Suppose then that $x^{\prime}$ extends $p$.

Note on the other hand that $h_{\text {Odd }}$ is not an extension of $p$, since it is a branch through $\operatorname{Lnbd}(p) \cup \operatorname{sInSeg}(p)$, which has no nodes extending $p$.

Thus, $h_{O d d}$ and $x^{\prime}$ disagree at a point before $\operatorname{lh}(p)=2 k \leq 2 e$. By induction it follows that there is $d, d^{\prime}, i, i^{\prime}$ so that $G\left(h_{O d d} \uparrow d, i\right)$ and $G\left(x^{\prime} \uparrow d^{\prime}, i^{\prime}\right)$ are both defined and are incompatible.

Recall that $x=p \frown\langle-n\rangle \frown h$. Let $\hat{d}=2 k+1+2 d$, so that $x \mid \hat{d}=p \frown\langle-n\rangle \frown$ $\left(h_{\text {Even }}\left\lceil d \times h_{\text {Odd }}\lceil d)\right.\right.$. The definition of $G$ is such that $G\left(x\lceil\hat{d}, i+1)=G\left(h_{\text {Odd }}\lceil d, i)\right.\right.$. Thus $G(x \uparrow \hat{d}, i+1)$ is defined and incompatible with $G\left(x^{\prime} \uparrow d^{\prime}, i^{\prime}\right)$. So $\hat{d}, d^{\prime}, \hat{i}=$ $i+1, i^{\prime}$ witness the condition in Claim 2.6, completing the inductive proof.

COROllary 2.7. $U$ has at most one branch.

Proof. Suppose $x$ and $x^{\prime}$ are two distinct branches of $U$. Let $d, d^{\prime}, i, i^{\prime}$ be given by Claim 2.6, so that $s=G\left(x\lceil d, i)\right.$ and $s^{\prime}=G\left(x^{\prime}\left\lceil d^{\prime}, i^{\prime}\right)\right.$ are defined and incompatible.

We prove that there are $\hat{s}$ extending $s$, and $\hat{s}^{\prime}$ extending $s^{\prime}$, so that there are branches through both $\mathcal{F}(\hat{s})$ and $\mathcal{F}\left(\hat{s}^{\prime}\right)$. Since $\hat{s}$ and $\hat{s}^{\prime}$ are incompatible (being extensions of the incompatible $s$ and $s^{\prime}$ ), this contradicts condition (2) in Claim 2.2 .

Let us prove that $s$ can be extended to $\hat{s}$ so that $\mathcal{F}(\hat{s})$ has a branch. The proof for $s^{\prime}$ is similar.

Look at $G(x\lceil e, i)$ and $H(x\lceil e, i)$ for $e \geq d$. By condition (4) in Claim 2.5 they are all defined. 
Suppose first that for all $e \geq d, H(x\lceil e, i)=*$. Then, using Claim 2.5, $\bigcup_{e \geq d} G\left(x\lceil e, i)\right.$ is a branch through $S^{*}$. Since the branch extends $s$, it follows by condition (1) in Claim 2.2 that there is a branch through $\mathcal{F}(s)$. We take $\hat{s}=s$ in this case.

Suppose on the other hand that for some $e \geq d, H(x\lceil e, i) \neq *$, and pick the least such $e$. Again using Claim 2.5, G(x「e,i) extends $s$, and $\bigcup_{j \geq e} H(x\lceil j, i)$ is a branch through $\mathcal{F}(G(x\lceil e, i))$. We take $\hat{s}=G(x\lceil e, i)$ in this case.

We have now established all the motivational properties of $U$ listed above. It remains to show, using the claims establishing these properties, that an application of INDEC to $U$ produces a branch through $S^{*}$.

Claim 2.8. $U$ is scattered.

Proof. Suppose for contradiction that $\pi$ embeds $\mathbb{Q}$ into $U$. We shall use $\pi$ to construct two branches through $U$, contradicting Corollary 2.7.

First, we divide $U$ into two parts. Let $p_{0}=\pi(0)$. Let $U_{1}=\left\{p \in U \mid p<_{L} p_{0} \vee p\right.$ is an initial segment of $\left.p_{0}\right\}$. Let $U_{2}=\left\{p \in U \mid p>_{L} p_{0}\right\}$. (The initial segments of $p_{0}$ are added to $U_{1}$ to make it a tree. The addition is not needed in the case of $U_{2}$.) We shall use the fact that $\pi$ embeds an interval of rationals into $U_{1}$, to produce a branch through it. A similar argument produces a branch through $U_{2}$. Since the two trees have no branches in common, we get two distinct branches of $U$, and hence the desired contradiction to Corollary 2.7.

Montalbán [4, Lemma 1.16] shows how to obtain a branch through a tree, starting from an embedding of the rationals into the Brouwer-Kleene order on the tree, working in $\mathrm{RCA}_{0}$. For completeness we sketch the argument.

First observe that:

(i) For every node $p, \pi$ embeds an interval into $U_{1} \cap \operatorname{nbd}(p)$ iff there are two distinct rationals sent into $U_{1} \cap \operatorname{nbd}(p)$ by $\pi$.

(ii) If $\pi$ embeds an interval into $\operatorname{nbd}(p)$, then there is an immediate extension $p^{-}\langle n\rangle$ of $p$ in $U$ so that $\pi$ sends two distinct rationals into $\operatorname{nbd}\left(p^{-}\langle n\rangle\right)$.

The first item follows from the fact that $\pi$ preserves order. The second item follows from preservation of order and the fact that $\left\{U \cap \operatorname{nbd}\left(p^{-}\langle n\rangle\right) \mid n \in I\right\}$ divides $\operatorname{nbd}(p)$ into a scattered collection of neighborhoods (it is ordered by $<_{I}$ of Diagram 1). The map into this collection induced by $\pi$ is order preserving from an interval of rationals, so it cannot be one-to-one.

Using (i) and (ii) it is easy to recursively construct a sequence $\emptyset=q_{0} \subsetneq q_{1} \ldots$ of nodes forming a branch through $U_{1}$. Pick at each stage $i$ the minimal pair of rational $a, a^{\prime}$-minimal in some recursive ordering of the rationals of order type $\omega$-so that $\pi(a)$ and $\pi\left(a^{\prime}\right)$ both belong to $\operatorname{nbd}\left(q_{i} \frown\langle n\rangle\right)$ for the same $n$. Set $q_{i+1}=q_{i} \frown\langle n\rangle$ for this $n$.

Claim 2.9. $U$ is not indecomposable to the left, and not indecomposable to the right.

Proof. Suppose for contradiction and definitiveness that $U$ is indecomposable to the left. (The argument for the right is similar.)

Let $y_{1}^{*}$ be the unique branch through $S_{1}^{*}$. Let $s=y_{1}^{*}\lceil 1$, let $p=p(s)$, and consider the node $p^{-}\langle-1\rangle$. 
Since $U$ is indecomposable to the left by assumption, there is an embedding $\pi_{L e f t}$ of $U$ into the $<_{L}$ interval of $U$ to the left of this node, namely into $\{q \in$ $U \mid q<_{L} p\ulcorner\langle-1\rangle\}$.

But by Claim 2.4 there is also an embedding $\pi_{\text {Right }}$ of $U$ into the right tail-end $\left\{q \in U \mid q>_{L} p^{\frown}\langle-1\rangle\right\}$.

Let $W$ be the complete binary tree, with order determined by $s \frown\langle 0\rangle\urcorner t<_{W} s$ and $s \frown\langle 1\rangle-t>_{W} s$. Using the two embeddings $\pi_{\text {Left }}$ and $\pi_{\text {Right }}$, one mapping to the left of $p \frown\langle-1\rangle$ and the other to the right, it is easy to construct an embedding $\sigma$ of $W$ into $U$. For example, set $\sigma(\emptyset)=p \frown\langle-1\rangle, \sigma(\langle 0\rangle\urcorner t)=\pi_{\text {Left }}(\sigma(t))$, and $\sigma(\langle 1\rangle-t)=\pi_{\text {Right }}(\sigma(t))$. Since $\mathbb{Q}$ embeds into $W$ it follows that $U$ is not scattered, contradicting Claim 2.8.

At last we are in a position to apply INDEC to the order $U$. Since $U$ is scattered, not indecomposable to the left, and not indecomposable to the right, it follows from INDEC that $U$ must be decomposable. In other words there must exist a cut $\langle A, B\rangle$ in $U$, so that $U$ embeds into neither $A$ nor $B$.

We use this cut to construct a branch through $S^{*}$. Call a node $s \in S^{*}$ nice if $p(s) \frown\langle-1\rangle \in A$ and $p(s) \frown\langle+1\rangle \in B$. We show that the nice nodes form a branch through $S^{*}$.

Claim 2.10. For every $k<\omega$, there is at most one nice $s \in S^{*}$ of length $k$.

Proof. Suppose for contradiction there are two distinct nice nodes $s$ and $s^{\prime}$ of length $k$. Suppose for definitiveness that $p(s)<_{L} p\left(s^{\prime}\right)$. So $q=p(s)-\langle+1\rangle<_{L}$ $p\left(s^{\prime}\right)^{\frown}\langle-1\rangle=q^{\prime}$, but then it cannot be that $q \in B$ and $q^{\prime} \in A$.

Claim 2.11. If $s \subsetneq s^{\prime}$ and $s^{\prime}$ is nice, then $s$ is nice.

Proof. Suppose $s \subsetneq s^{\prime}$ and $s^{\prime}$ is nice. Let $q=p(s) \frown\langle-1\rangle$ and $q^{\prime}=p\left(s^{\prime}\right) \frown\langle-1\rangle$. Let $2 k=\operatorname{lh}(p(s))<\operatorname{lh}\left(p\left(s^{\prime}\right)\right)$. Then $q(2 k)=-1<_{I} 0=q^{\prime}(2 k)$, so $q<_{L} q^{\prime}$. Since $q^{\prime} \in A$ it follows from this that $q \in A$. A similar argument shows $p(s) \frown\langle+1\rangle \in B$.

Claim 2.12. For every $k<\omega$, there exists a nice $s$ of length $k$.

Proof. By Claim 2.2, there exists $s$ of length $k$ so that $\mathcal{F}(s)$ has a branch. We claim that this $s$ is nice.

Let $p=p(s)$. By Claim 2.4, $U$ embeds into the left tail-end $\left\{q \in U \mid q<_{L}\right.$ $\left.p^{\frown}\langle+1\rangle\right\}$. If $p^{\frown}\langle+1\rangle \in A$ then $A$, which is closed leftward, contains this left tail-end, and hence $U$ embeds into $A$. But this contradicts the fact that $\langle A, B\rangle$ is a decomposition of $U$. So $p \frown\langle+1\rangle$ must belong to $B$. A similar argument using the fact that $U$ embeds into $\left\{q \in U \mid q>_{L} p^{\frown}\langle-1\rangle\right\}$ shows that $p^{\frown}\langle-1\rangle$ must belong to $A$.

It is now easy to complete the proof that there is a branch through $S^{*}$, and with it the proof of the instance of weak $\Sigma_{1}^{1}$ choice we are working on.

Let $R=\left\{s \in S^{*} \mid p(s) \frown\langle-1\rangle \in A \wedge p(s)^{\frown}\langle\langle+1\rangle \in B\}\right.$. That $R$ exists follows easily from the existence of $A$ and $B$ using $\Delta_{1}^{0}$ comprehension. By Claims 2.10 and 2.11, $s(i)=m$ for some $s \in R$ iff $s(i)=m$ for all $s \in R$ with $\operatorname{lh}(s)>i$. So the set $y^{*}=\{\langle i, m\rangle \mid s(i)=m$ for some $s \in R\}$ exists, again using $\Delta_{1}^{0}$ comprehension, and moreover it is a function. By Claims 2.12 the domain of $y^{*}$ 
is $\omega$. For each $k<\omega, y^{*}\lceil k$ is an element of $R$ by definition, and therefore also an element of $S^{*}$. So $y^{*}$ is a branch through $S^{*}$.

By the definition of $S^{*}$, its branch $y^{*}$ must have the form $\Pi_{n<\omega} y_{n}$ with $y_{n} \in S_{n}$, equivalently $\varphi\left(n, y_{n}\right)$, for each $n$. This completes the proof of the existence of a sequence $\left\langle y_{n} \mid n<\omega\right\rangle$ so that $(\forall n) \varphi\left(n, y_{n}\right)$.

§3. A model of weak $\Sigma_{1}^{1}$ choice in which INDEC fails. Steel $[7,8]$ developed a powerful technique for creating models of hyperarithmetic analysis. The technique was used by Steel to produce a model of $\Delta_{1}^{1}$ comprehension where $\Sigma_{1}^{1}$ choice fails, showing that the former does not imply the latter. Van Wesep [9] in another application produced a model of weak $\Sigma_{1}^{1}$ choice where $\Delta_{1}^{1}$ comprehension fails. We prove that INDEC fails in Van Wesep's model. It follows that weak $\Sigma_{1}^{1}$ choice does not imply INDEC.

Let us briefly recall Steel's technique and Van Wesep's model. We follow Van Wesep's exposition. The poset he uses differs slightly from the one Steel used, allowing non-wellfounded "ordinals" as tags.

Let $\prec$ be a recursive (illfounded) linear order on a recursive subset of $\omega$, so that the wellfounded part of $\prec$ has order type $\omega_{1}^{\text {ck }}$, and so that no hyperarithmetic sequence witnesses the illfoundedness of $\prec$.

Define a poset $\mathbb{P}$ as follows. Conditions are triples $p=\left\langle T_{p}, f_{p}, h_{p}\right\rangle$ where:

1. $T_{p} \subseteq \omega^{<\omega}$ is a finite tree.

2. $f_{p}$ is a function from a finite subset of $\omega$ to $T$. Let $\operatorname{Dc}\left(f_{p}\right)$, the downward closure of range $\left(f_{p}\right)$, be the set $\left\{f_{p}(i)\left\lceil j \mid i \in \operatorname{dom}\left(f_{p}\right), j \leq \operatorname{lh}\left(f_{p}(i)\right)\right\}\right.$ of initial segments of nodes in range $\left(f_{p}\right)$.

3. $h_{p}$ is a $\left\langle T_{p}, f_{p}\right\rangle$-tagging. I.e., $h_{p}$ is a function from $T_{p}-\{\emptyset\}-\operatorname{Dc}\left(f_{p}\right)$ into $\operatorname{dom}(\prec)$, with $t \supsetneq s \rightarrow h_{p}(t) \prec h_{p}(s)$.

Conditions are ordered by reverse extension. $p \leq q$ iff $T_{p} \supseteq T_{q}, h_{p} \supseteq h_{q}$, and $\left(\forall i \in \operatorname{dom}\left(f_{q}\right)\right) f_{p}(i)$ is defined and extends $f_{q}(i)$.

We use $\mathbb{P}$ to force over the model $L_{\omega_{1}^{c k}}$. Let $G$ be generic over this model. Let $T=T^{G}=\bigcup_{p \in G} T_{p}$ and define $h=h^{G}$ and $f(i)=f^{G}(i)$ similarly. We use $\dot{T}, \dot{f}$, and $\dot{h}$ for the canonical names for $T, f$, and $h$. T is a tree on $\omega$, $\mathcal{B}=\mathcal{B}^{G}=\{f(i) \mid i \in \omega\}$ is a set of branches through $T$, and $h$ "ranks" nodes of $T$ which are not initial segments of branches in $\mathcal{B}$, meaning that it embeds the order of reverse extension on these nodes to $\prec$. (If $\prec$ were wellfounded then $h$ would witness that these nodes do not extend to branches of $T$.)

In talking about $h$, we identify each member of the wellfounded part of $\prec$ with its ordinal rank. Thus when we write $h(t)=\alpha$ we mean $h(t)=i$ for $i$ whose order type in $\prec$ is $\alpha$. For $t \in T$ so that $h(t)$ is defined we refer to $h(t)$ as the $\operatorname{tag}$ of $t$.

For each finite $F \subseteq \mathcal{B}$ let $M_{F}=M_{F}^{G}$ be the model $L_{\omega_{1}^{c k}}(\{T\} \cup F)$. The subsets of $\omega$ which belong to $M_{F}$ are precisely those which are hyperarithmetic in the join of $\{T\} \cup F$. The models of hyperarithmetic analysis that we produce are unions of models of the form $M_{F} \cap(\omega \cup \mathcal{P}(\omega))$.

$M_{F}$ has the tree $T$, but not the tagging function $h$. For each $\alpha<\omega_{1}^{\text {ck }}$, the restriction of $h$ to nodes with tags $<\alpha$ does belong to $M_{F}$ : genericity implies that the tag of $t$, when wellfounded, is precisely equal to the rank of $t$ in $T$, and for 
each $\alpha<\omega_{1}^{\text {ck }}$ the ranks up to $\alpha$ can be computed from $T$ by recursion. But these recursions become increasingly complicated as $\alpha$ increases. If one is restricted to some bounded complexity below hyperarithmetic, then one cannot distinguish between sufficiently high tags. A precise formulation of this symmetry is given in Lemma 3.3 below.

Let $A \subseteq \omega$ be finite. Let $F(A)=F^{G}(A)$ denote the set $\left\{f^{G}(i) \mid i \in A\right\}$. By induction on $\alpha<\omega_{1}^{c k}$ we define the $A$-nice names for elements of $L_{\alpha}(\{T\} \cup F(A))$, and the order of these names. The order of $\dot{x}$ is denoted $o(\dot{x})$, and we shall have $L_{\alpha}(\{T\} \cup F(A))=\{\dot{x}[G] \mid \dot{x}$ is $A$-nice and $o(\dot{x})<\alpha\}$. We start the hierarchy with $L_{\omega}(\{T\} \cup F(A))=L_{\omega} \cup\left\{T^{G}\right\} \cup F(A)$. $1_{\mathbb{P}}$ denotes $\langle\emptyset, \emptyset, \emptyset\rangle$, the weakest condition in $\mathbb{P}$.

- The $A$-nice names for elements of $L_{\omega}$, for $T^{G}$, and for $f^{G}(i), i \in A$, are simply the canonical $\mathbb{P}$-names for these objects. The order of these names is 0 .

- Let $\alpha \geq \omega$. Let $\dot{z}=\left\{\left\langle\dot{x}, 1_{\mathbb{P}}\right\rangle \mid \dot{x}\right.$ is $A$-nice and $\left.o(\dot{x})<\alpha\right\}$. (By induction, $\dot{z}$ names $L_{\alpha}(\{T\} \cup F(A))$.) If $\varphi\left(v_{0}, v_{1}, \ldots, v_{k}\right)$ is a formula, and $\dot{a}_{1}, \ldots, \dot{a}_{k}$ are $A$-nice names of order $<\alpha$, then $\{\langle\dot{u}, p\rangle \mid \dot{u}$ is $A$-nice, $o(\dot{u})<\alpha$, and $p \Vdash$ " $\left.\dot{z} \models \varphi\left[\dot{u}, \dot{a}_{1}, \ldots, \dot{a}_{k}\right] "\right\}$ is an $A$-nice name of order $\alpha$.

It is clear that every element of $M_{F}^{G}=L_{\omega_{1}^{c k}}\left(\{T\} \cup F^{G}(A)\right)$ has an $A$-nice name, and that $M_{F}^{G}=\{\dot{x}[G] \mid \dot{x}$ is $A$-nice $\}$.

A statement $\varphi\left(\dot{x}_{1}, \ldots, \dot{x}_{k}\right)$ in the forcing language is $A$-nice if $\dot{x}_{i}$ are $A$-nice, and all quantifiers of $\varphi$ are bounded to range over $A$-nice names. When talking about $M_{F(A)}^{G}$ in the forcing language we shall only use $A$-nice statements. We often neglect to mention explicitly that the statements are $A$-nice. $A$ will always be a finite set, and we often neglect to explicitly mention this too.

An $A$-nice statement $\varphi\left(\dot{x}_{1}, \ldots, \dot{x}_{k}\right)$ is ranked if there is $\alpha<\omega_{1}^{\text {ck }}$ so that $o\left(\dot{x}_{i}\right)<$ $\alpha$ and all quantifiers in $\varphi$ are bounded to range over $A$-nice names of order $<\alpha$. The least $\alpha$ witnessing this is the order of $\varphi\left(\dot{x}_{1}, \ldots, \dot{x}_{k}\right)$. The rank of $\varphi\left(\dot{x}_{1}, \ldots, \dot{x}_{k}\right)$ is defined to be $\omega^{2} \cdot o+\omega \cdot q+n$ where $o$ is the order of $\varphi\left(\dot{x}_{1}, \ldots, \dot{x}_{k}\right)$, $q$ is the number of quantifier in $\varphi$, and $n$ the number of logical connectives. The definition is taken from Steel [8].

Claim 3.1. For each $\alpha<\omega_{1}^{\mathrm{ck}}$, the restriction of the forcing relation to A-nice statements of rank $<\alpha$ belongs to $L_{\omega_{1}^{c k}}$.

Claim 3.1 is clear. It is taken from Van Wesep [9] and relies on Van Wesep's definition of $\mathbb{P}$, which differs slightly from that of Steel [8].

Definition 3.2. Let $p, p^{*} \in \mathbb{P}, \eta<\omega_{1}^{\mathrm{c} k} \cdot p^{*}$ is an $\eta$-absolute $A$-reduct of $p$ if:

1. $T_{p}=T_{p^{*}}$ and $f_{p}(i)=f_{p^{*}}(i)$ for $i \in A$.

2. If $h_{p}(s)<\eta$ then $h_{p^{*}}(s)=h_{p}(s)$. If $h_{p}(s) \geq \eta$ then $h_{p^{*}}(s) \geq \eta$.

In condition (2) we adopt the convention that $h_{p}(s)=\infty$ for $s \in \operatorname{Dc}\left(f_{p}\right)$, and that $\infty \geq \eta$.

Lemma 3.3 (Steel [8]). Let $\varphi\left(\dot{x}_{1}, \ldots, \dot{x}_{k}\right)$ be A-nice and ranked, with rank $\leq$ $\eta<\omega_{1}^{\text {ck }}$. Suppose $p^{*}$ is an $\omega \eta$-absolute A-reduct of $p$. Then $p \Vdash \varphi\left(\dot{x}_{1}, \ldots, \dot{x}_{k}\right)$ iff $p^{*} \Vdash \varphi\left(\dot{x}_{1}, \ldots, \dot{x}_{k}\right)$. 
Lemma 3.3 is the foundation of Steel's method for reasoning about the models of hyperarithmetic analysis that he produces. It shows in a very precise way that if one is restricted to complexity bounded below hyperarithmetic, in $T$ and finitely many branches through it, then one cannot distinguish the tags of nodes in $T$ beyond a bounded level. It implies in particular that the only branches of $T$ in $M_{F}^{G}$ are the ones in $F$ :

Claim 3.4 (Steel [8]). Let $A \subseteq \omega$ be finite. Let $F=\left\{f^{G}(i) \mid i \in A\right\}$. Then the only branches of $T$ which belong to $M_{F}^{G}$ are those in $F$.

Proof. Suppose not. Let $\dot{b}$ be an $A$-nice name for a branch of $T$ which is distinct from $f^{G}(i)$ for each $i \in A$. Let $p \in \mathbb{P}$ force this. Strengthening $p$, we may fix $n<\omega$ and a node $t$, and assume that $p$ forces $\dot{b} \mid \check{n}=\check{t}, t \in T_{p}$, and $t$ is incompatible with $f_{p}(i)$ for each $i \in A$.

Let $\eta<\omega_{1}^{\text {ck }}$ be the rank of the statement " $\dot{b}$ is a branch through $\dot{T}$, and $\dot{b}\lceil\check{n}=\check{t} . "$ (How large it is exactly depends on the order of $\dot{b}$.)

The key to the proof is our ability to change the value of $\dot{h}(\check{t})$, from $h_{p}(t)$ which possibly belongs to the illfounded part of $\prec$, to a new value $h_{p^{*}}(t)$ which is in the wellfounded part, without affecting the statement that $\dot{b}$ is a branch of $\dot{T}$ extending $\check{t}$.

Precisely, let $p^{*}$ be obtained from $p$ by setting $T_{p^{*}}=T_{p}$, setting $f_{p^{*}}=f_{p} \uparrow A$, picking wellfounded values $\geq \omega \eta$ for $h_{p^{*}}(s)$ for all $s \in T_{p}-\operatorname{Dc}\left(f_{p^{*}}\right)$ so that $h_{p}(s)$ is undefined or $\geq \omega \eta$, and leaving $h_{p^{*}}(s)=h_{p}(s)$ for all other $s$. Then $p^{*}$ is an $\omega \eta$-absolute $A$-reduct of $p$. Since $t$ is incompatible with $f_{p}(i)$ for each $i \in A, t$ does not belong to $\operatorname{Dc}\left(f_{p^{*}}\right)$. Therefore $h_{p^{*}}(t)$ is defined, and by construction of $p^{*}, h_{p^{*}}(t)$ belongs to the wellfounded part of $\prec$. By Lemma $3.3, p^{*}$ forces that $\dot{b}$ is a branch through $\dot{T}$, and $\dot{b}$ extends $\check{t}$. But then $\dot{h}\left(\dot{b}\lceil\breve{j}), j>n\right.$, is forced by $p^{*}$ to be a descending chain in $\prec$ below $\dot{h}(\check{t})=h_{p^{*}}(t)$, contradiction.

The models of analysis that we construct, just like the models in Steel [8] and Van Wesep [9], are all of the form $(\omega \cup \mathcal{P}(\omega)) \cap N_{K}$ where $N_{K}=\bigcup_{F \subset K, F \text { finite }} M_{F}$, for $K \subseteq \mathcal{B}$. (The models constructed in Montalbán [4] and [3] are of similar form with a slightly different forcings, for example, in [4], designed to add a ranking function on an open game rather than a ranking function on a tree.) The parameter affecting the exact model we obtain is the set $K \subseteq \mathcal{B}$. All such models, regardless of the choice of $K$, satisfy RCA and indeed are model of hyperarithmetic analysis.

Clearly $T$ belongs to $N_{K}$, as it belongs to $M_{F}$ for each $F$. The branches of $T$ that belong to $K$ also belong to $N_{K}$. By Claim 3.4 these are the only branches of $T$ which belong to $N_{K}$. Moreover:

Claim 3.5 (Steel [8]). In $N_{K}$ there are no sequences $\left\langle b_{n} \mid n<\omega\right\rangle$ of infinitely many distinct branches through $T$.

Proof. Suppose $\left\langle b_{n} \mid n<\omega\right\rangle$ belongs to $N_{K}$. Then there is a finite $F \subseteq K$ so that $\left.\left\langle b_{n}\right| n<\omega\right\}$ belongs to $M_{F}$. But then, since $\left\{b_{n}|n<\omega\rangle\right.$ is infinite and $F$ is finite, there must be a branch $b_{n}$ of $T$ in $M_{F}$ which does not belong to $F$, contradicting Claim 3.4. 
Steel [8] uses this claim to argue that $\Sigma_{1}^{1}$ choice fails in $N_{K}$ for $K=\mathcal{B}$ : by genericity, $(\forall n)(\exists b)(b$ is a branch of $T$ and $b(0)>n)$. But by the claim there is no choice function inside $N_{K}$ for this $\Sigma_{1}^{1}$ statement.

Van Wesep [9] also uses the claim. He carefully selects $K$ so that there is a real which codes a sequence of infinitely many distinct branches of $T$, and is $\Delta_{1}^{1}(T)$ over $N_{K}$. By the claim the real cannot belong to $N_{K}$, and it follows that $\Delta_{1}^{1}$ comprehension fails in $N_{K}$. On the other hand Van Wesep proves that weak $\Sigma_{1}^{1}$ choice holds in the model.

We use Van Wesep's model to prove that weak $\Sigma_{1}^{1}$ choice does not imply INDEC. After describing the model (i.e., describing the set $K$ ), we shall show that INDEC fails in it.

Let $(*, *): \omega \times \omega \rightarrow \omega$ be a recursive injection of $\omega^{2}$ into $\omega$, with the property that $(m, n)>m$ for all $m, n$. Let $\pi: \omega^{<\omega} \rightarrow \omega$ be a recursive injection, with the property that $\pi(\emptyset)>0$ and $\pi(t)>t(0)$ for all $t \neq \emptyset$.

By recursion on $l<\omega$ define $m_{l} \in \omega$ and $D_{l} \subseteq \omega$ as follows:

- $m_{0}=0$, and $D_{0}=\{\pi(b\lceil i) \mid b \in \mathcal{B}, i \in \omega$, and $(\exists n) b(0)=(0, n)\}$.

- $m_{l+1}$ is the least $m>m_{l}$ with $m \notin D_{l}$. $D_{l+1}=D_{l} \cup\{\pi(b \mid i) \mid b \in \mathcal{B}, i \in \omega$, and $\left.(\exists n) b(0)=\left(m_{l+1}, n\right)\right\}$.

Let $D=\bigcup_{l<\omega} D_{l}$. The following properties are clear:

1. $D_{l}$ omits infinitely many numbers, so $m_{l+1}$ can be defined for each $l$.

2. By genericity, for each $m$ there are infinitely many $n$ so that $(\exists b \in \mathcal{B}) b(0)=$ $(m, n)$.

3. All elements of $D_{0}$ are greater than 0 , and all elements of $D_{l+1}-D_{l}$ are greater than $m_{l+1}$. This uses the particular nature of the injection $\pi$ picked above.

It follows that for all $l, m_{l} \notin D$. On the other hand $\left\{m_{l} \mid l<\omega\right\} \supseteq \omega-D$ by the definition of $m_{l+1}$. So:

4. $\left\{m_{l} \mid l<\omega\right\}=\omega-D$.

Let $K=\{b \in \mathcal{B} \mid(\exists m, n)(b(0)=(m, n) \wedge m \notin D\}$. We work with this specific $K$ for the rest of the section. By $(4), K=\left\{b \in \mathcal{B} \mid(\exists l, n) b(0)=\left(m_{l}, n\right)\right\}$. From this and the definition of $D_{l}$ it follows that:

5. $D=\{\pi(b\lceil i) \mid i<\omega \wedge b \in K\}$.

Since the branches of $T$ which belong to $N_{K}$ are precisely the ones in $K$, it follows from the above properties that $D$ is $\Delta_{1}^{1}(T)$ over $N_{K}: j \in D$ iff $j=\pi(t)$ for $t$ which can be extended to a branch of $T$ in $N_{K}$ by (5); and $j \notin D$ iff there is a branch $b$ of $T$ in $N_{K}$ with $b(0)=(j, n)$ for some $n$, by (2) and the definition of $K$. It also follows from condition (5) that from $D$ one can construct infinitely many branches of $T$, so by Claim $3.5, D \notin N_{K}$.

The definition of $D$, its properties, the definition of $K$, and the claim above are all taken form Van Wesep [9]. Van Wesep also proves the following lemma. He uses it, together with the fact that $D$ is $\Delta_{1}^{1}(T)$ over $N_{K}$ and does not belong to $N_{K}$, to conclude that weak $\Sigma_{1}^{1}$ choice does not imply $\Delta_{1}^{1}$ comprehension.

Lemma 3.6 (Van Wesep [9]). $N_{K}$ satisfies weak $\Sigma_{1}^{1}$ choice.

Proof Sketch. Suppose $\varphi(x)$ is arithmetic in parameters from $M_{F}$. Suppose that there exists $x \in N_{K}-M_{F}$ so that $\varphi(x)$ holds. A forcing symmetry argument 
shows that there must then exist two (in fact infinitely many) distinct witnesses $x$ for $\varphi(x)$ in $N_{K}$. One of the keys to the argument is the fact $N_{K}$ can be viewed as an extension of $M_{F}$, adding the branches of $T$ which belong to $K-F$. Another is that any node which is an initial segment of such a branch, is an initial segment of two (in fact infinitely many) different branches in $K-F$. This is but a hint to the proof. For more see [9]. The main subtlety is in the first fact above, which only applies to ranked forcing statements, and uses Lemma 3.3.

Suppose now that $\psi(n, x)$ is arithmetic in parameters from $N_{K}$ and for every $n$ there is a unique $x=x_{n} \in N_{K}$ so that $\psi(n, x)$. Fix $F \subseteq K$ finite so that the parameters of $\psi$ all belong to $M_{F}$. Then by the previous paragraph it must be that $x_{n} \in M_{F}$ for each $n$. The model $M_{F}$ satisfies weak $\Sigma_{1}^{1}$ choice, so the sequence $\left\langle x_{n} \mid n<\omega\right\rangle$ belongs to $M_{F}$, and hence also to $N_{K}$.

Our goal here is to show that weak $\Sigma_{1}^{1}$ choice does not imply INDEC. All we need is the following lemma (for Van Wesep's set $K$ described above):

LEMma 3.7. INDEC fails in $N_{K}$.

Proof. We define a tree $S^{*}$ so that the unique branch through $S^{*}$ codes the set $D$, and define a map $\mathcal{F}$ so that $S^{*}$ and $\mathcal{F}$ have the properties in Claim 2.2. The results of Section 2 then show that from INDEC one can derive the existence of $D$. But $D \notin N_{K}$, so INDEC fails in $N_{K}$.

let $W=\left\{t \in \omega^{<\omega} \mid \pi(t) \in D\right\}$. We use the following properties of $W$, which follow from the properties of $D$ above:

(a) $W \subseteq T$ and $W$ has no terminal nodes.

(b) If $t \notin W$, then there is a node of length 1 in $W$ of the form $\langle(\pi(t), n)\rangle$.

(c) The converse of (b) is also true. If there is a node of length 1 in $W$ of the form $\langle(\pi(t), n)\rangle$, then $t \notin W$.

(d) $t \in W$ iff it $t$ can be extended to a branch of $T$ in $K$.

Let $S^{*}$ be the tree of attempts to construct $\chi: T \rightarrow 2$ which is a characteristic function of a tree, and $r: T \rightarrow T$ witnessing in a uniquely determined manner that this tree has properties (a) and (b). Precisely:

(i) $\chi$ is the characteristic function of a tree $\chi^{-1}(1) \subseteq T$.

(ii) For each $t \in \chi^{-1}(1), r(t)$ is an immediate extension of $t$ in $\chi^{-1}(1)$, and the left-most such.

(iii) For each $t \notin \chi^{-1}(1), r(t)$ is a node of length 1 in $\chi^{-1}(1)$ so that $r(t)(0)$ has the form $(\pi(t), n)$, with $n$ least so that a node of this form belongs to $\chi^{-1}(1)$.

Even more precisely, let $\left\{t_{i} \mid i<\omega\right\}$ enumerate $T$, with the property that initial segments of $t$ are enumerated before $t$. A node $s$ of length $k$ in $S^{*}$ consists of functions $\chi=\chi^{s}:\left\{t_{0}, \ldots, t_{k-1}\right\} \rightarrow 2$ and $r=r^{s}:\left\{t_{0}, \ldots, t_{k-1}\right\} \rightarrow \omega^{<\omega}$ satisfying the conditions:

- $\chi\left(t_{i}\right) \in\{0,1\}$ for each $i$, and $\chi^{-1}(1)$ is closed under initial segments.

- If $i<k$ and $\chi\left(t_{i}\right)=1$ then $r\left(t_{i}\right)$ is an immediate extension of $t_{i}$. Let $n$ be such that $r\left(t_{i}\right)=t_{i} \frown\langle n\rangle$. For $j<k$ such that $t_{j}$ has the form $t_{i} \frown\langle\bar{n}\rangle$, if $\bar{n}<n$ then $\chi\left(t_{j}\right)=0$, and if $\bar{n}=n$ then $\chi\left(t_{j}\right)=1$. 
- If $i<k$ and $\chi\left(t_{i}\right)=0$ then $r\left(t_{i}\right)$ has the form $\left\langle\left(\pi\left(t_{i}\right), n\right)\right\rangle$ for some $n$. For $j<k$ such that $t_{j}$ has the form $\left\langle\left(\pi\left(t_{i}\right), \bar{n}\right\rangle\right.$, if $\bar{n}<n$ then $\chi\left(t_{j}\right)=0$, and if $\bar{n}=n$ then $\chi\left(t_{j}\right)=1$.

We shall refer to $s$ as equal to $\chi^{s} \times r^{s}$ for notational simplicity, but really $s$ is equal to $\left\langle\left(\chi^{s}\left(t_{i}\right), \pi\left(r^{s}\left(t_{i}\right)\right)|i<k\rangle\right.\right.$, so that $S^{*}$ is formally a tree on $\omega$.

Claim 3.8. There are no branches of $S^{*}$ in $N_{K}$.

Proof. This is essentially the proof that $D$ does not belong to $N_{K}$.

Suppose $b$ is a branch through $S^{*}$. Then $b$ gives rise to functions $\chi$ and $r$ with properties (i)-(iii) above. Consider the tree $\chi^{-1}(1)$. It is a subtree of $T$ by property (i). By property (ii) it has no terminal nodes. Since $T$ has infinitely many terminal nodes it follows that $T-\chi^{-1}(1)$ is infinite, and by property (iii) it follows that $\chi^{-1}(1)$ has infinitely many distinct nodes of length 1 . They can constructively be extended to branches through the tree, since the tree has no terminal nodes. All this can be done inside $N_{K}$. Since $\chi^{-1}(1) \subseteq T$ it follows that, in $N_{K}$, one can construct a sequence of infinitely many distinct branches of T. But this contradicts Claim 3.5.

A node $s=\chi^{s} \times r^{s} \in S^{*}$ of length $k$ determines more of $\chi$ than its restriction to $k$, because of properties (ii) and (iii) above. The full information that $s$ gives on $\chi$ is captured by the partial map $\theta^{s}: T \rightarrow\{0,1\}$ defined as follows:

- If $i<k$ then $\theta^{s}\left(t_{i}\right)=\chi^{s}\left(t_{i}\right)$.

- If $i<k, \chi^{s}\left(t_{i}\right)=1$, and $r^{s}\left(t_{i}\right)=t_{i} \frown\langle n\rangle$, then $\theta^{s}\left(t_{i} \frown\langle n\rangle\right)=1$ and $\theta^{s}\left(t_{i} \frown\langle\bar{n}\rangle\right)=0$ for all $\bar{n}<n$.

- If $i<k, \chi^{s}\left(t_{i}\right)=0$, and $r^{s}\left(t_{i}\right)=\left\langle\left(\pi\left(t_{i}\right), n\right)\right\rangle$, then $\theta^{s}\left(\left\langle\left(\pi\left(t_{i}\right), n\right)\right\rangle\right)=1$ and $\theta^{s}\left(\left\langle\left(\pi\left(t_{i}\right), \bar{n}\right)\right\rangle\right)=0$ for all $\bar{n}<n$.

It is clear from the definitions that if $\chi$ is the characteristic function determined by a branch of $S^{*}$ extending $s$, then $\chi$ extends $\theta^{s}$.

For $t$ such that $\theta^{s}(t)=1$, let $V_{t}^{s}$ be the tree of attempts to construct a branch of $T$ extending $t$. Precisely, $V_{t}^{s}=T(t)$. For $t$ such that $\theta^{s}(t)=0$, let $V_{t}^{s}$ be the tree of attempts to construct a branch $b$ of $T$ with $b(0)$ of the form $\langle(\pi(t), n)\rangle$. Precisely, $V_{t}^{s}$ consists of all nodes of $T$ with first coordinate of this form, plus the empty node.

The following claim is then obvious from the definitions, properties (c) and (d) above, and the fact that the branches of $T$ which belong to $N_{K}$ are precisely the ones in $K$.

Claim 3.9. If $\theta^{s}(t)=1$ and there is a branch of $V_{t}^{s}$ in $N_{K}$, then $t \in W$. If $\theta^{s}(t)=0$ and there is a branch of $V_{t}^{s}$ in $N_{K}$, then $t \notin W$.

For each $s \in S^{*}$ define $\mathcal{F}(s)$ to be the tree $\Pi_{t \in \operatorname{dom}\left(\theta^{s}\right)} V_{t}^{s}$.

Claim 3.10. Let $s, s^{\prime} \in S^{*}$ be incompatible. Then at most one of $\mathcal{F}(s), \mathcal{F}\left(s^{\prime}\right)$ has a branch in $N_{K}$.

ProOF. From the fact that $s$ and $s^{\prime}$ are incompatible, it follows that $\theta^{s}$ and $\theta^{s^{\prime}}$ are incompatible. (The last two conditions in the definition of $\theta^{s}$ are essential here, in case that $s$ and $s^{\prime}$ disagree only on their $r$ parts.) Let $t \in \operatorname{dom}\left(\theta^{s}\right) \cap$ $\operatorname{dom}\left(\theta^{s^{\prime}}\right)$ be such that $\theta^{s}(t) \neq \theta^{s^{\prime}}(t)$. A branch of $\mathcal{F}(s)$ includes a branch through 
$V_{t}^{s}$, and similarly with $s^{\prime}$. If there are branches through both $V_{t}^{s}$ and $V_{t}^{s^{\prime}}$ in $N_{K}$, then since $\left\{\theta^{s}(t), \theta^{s^{\prime}}(t)\right\}$ includes both 1 and 0 , it follows by the last claim that $t \in W$ and $t \notin W$, a contradiction.

Claim 3.11. For each $k$ there is some $s \in S^{*}$ of length $k$ so that $\mathcal{F}(s)$ has a branch in $N_{K}$.

Proof. Fix $k$. For $i<k$ set $\chi^{s}\left(t_{i}\right)=1$ if $t_{i} \in W$ and $\chi^{s}\left(t_{i}\right)=0$ if $t_{i} \notin W$. If $t_{i} \in W$ then using (a) above let $n$ be least so that $t_{i} \frown\langle n\rangle \in W$, and set $r^{s}\left(t_{i}\right)=$ $t_{i} \frown\langle n\rangle$. If $t_{i} \notin W$ then using (b) above let $n$ be least so that $\left\langle\left(\pi\left(t_{i}\right), n\right)\right\rangle \in W$ and set $r^{s}\left(t_{i}\right)=\left\langle\left(\pi\left(t_{i}\right), n\right)\right\rangle$. Finally let $s=\chi^{s} \times r^{s}$. It is clear from this definition that $\theta^{s}(t)=1$ iff $t \in W$ for each (of the finitely many) $t \in \operatorname{dom}\left(\theta^{s}\right)$. From this and properties (b) and (d) above it follows that each of the trees $V_{t}^{s}, t \in \operatorname{dom}\left(\theta^{s}\right)$, has a branch in $K$ and therefore in $N_{K}$. So $\mathcal{F}(s)$ has a branch in $N_{K}$.

Both $S^{*}$ and $\mathcal{F}$ belong to $N_{K}$. In fact they belong to $M_{\emptyset}^{G}$, as both are defined from $T$. We established that, in $N_{K}$, they have the properties derived in Claim 2.2. (The first condition in the claim holds for the current $S^{*}$ vacuously, since in $N_{K}$ no $s \in S^{*}$ can be extended to a branch of $S^{*}$.)

Recall that in Section 2 we proved, from the properties given by Claim 2.2, using INDEC, that there is a branch through $S^{*}$.

Suppose for contradiction that INDEC is true in $N_{K}$. Then the proof in Section 2, applied with the current $S^{*}$ and $\mathcal{F}$ and relativized to the model $N_{K}$, shows that there is a branch of $S^{*}$ in $N_{K}$. But this contradicts Claim 3.8. The contradiction completes the proof of Lemma 3.7.

We have now shown that (in the base theory RCA) weak $\Sigma_{1}^{1}$ choice does not imply INDEC. The model $N_{K} \cap(\omega \cup \mathcal{P}(\omega))$ satisfies the former by Lemma 3.6 and fails to satisfy the latter by Lemma 3.7 .

\$4. A model of INDEC in which $\Delta_{1}^{1}$ comprehension fails. We continue to work with the poset $\mathbb{P}$ of the previous section. Fix $G$ which is generic for this poset over $L_{\omega_{1}^{c k}+1}$. For the most part we work only with ranked forcing statements, so that we have access to Lemma 3.3. In those situations genericity over $L_{\omega_{1}^{c k}}$ is enough. But every once in a while, when dealing with statements which are not $\Delta_{1}$ over $L_{\omega_{1}^{c k}}[G]$, for example an order being scattered in $M_{F}^{G}$, we implicitly use the fact that $G$ meets dense sets outside $L_{\omega_{1}^{\text {ck }}}$.

We work with $T=T^{G}, f(i)=f^{G}(i), \mathcal{B}=\mathcal{B}^{G}, h=h^{G}$, and $M_{F}=M_{F}^{G}$ all defined as before. Recall that $\dot{T}, \dot{f}, \dot{\mathcal{B}}$, and $\dot{h}$ name these objects. Given a finite $A \subseteq \omega$ we set $M_{F(A)}^{G}=M_{F}^{G}=L_{\omega_{1}^{c k}}(\{T\} \cup F)$ where $F=\left\{f^{G}(i) \mid i \in A\right\}$. We use $\dot{M}_{F(A)}$ for the canonical name for this model. This is a class name over $L_{\omega_{1}^{\text {ck }}}$.

Let $\mathbb{C}$ be the poset adding a Cohen real. Conditions are finite partial functions from $\omega$ into 2 , ordered by reverse extension. Let $H$ be generic for $\mathbb{C}$ over $L_{\omega_{1}^{c k}+1}[G]$.

Set $K=\{b \in \mathcal{B} \mid b(0)=(n, e)$ with $e$ even if $H(n)=0$ and odd if $H(n)=1\}$. Let $\dot{K}=\left\{\langle\dot{f}(i),\langle p, c\rangle\rangle \mid f_{p}(i)(0)=(n, e)\right.$ with $e$ even iff $\left.c(n)=0\right\}$, so that $\dot{K}$ is a $\mathbb{P} \times \mathbb{C}$ name for $K$. Set $I=\left\{i<\omega \mid f^{G}(i) \in K\right\}$, and let $\dot{I}=\{\langle\check{i},\langle p, c\rangle\rangle \mid$ $f_{p}(i)(0)=(n, e)$ with $e$ even iff $\left.c(n)=0\right\}$, so that $\dot{I}$ names $I$. 
As in the previous section let $N_{K}=\bigcup_{F \subseteq K, F \text { finite }} M_{F}$. Let $\dot{N}_{K}$ be the natural name for $N_{K}$.

By genericity of $G$, for each $n$ there are infinitely many even numbers $e$, and infinitely many odd numbers $e$, so that $\langle(n, e)\rangle$ can be extended to a branch in $\mathcal{B}$. Thus $H(n)=0$ iff $(\exists b \in K)(\exists e \in \operatorname{Even}) b(0)=(n, e)$, and $H(n)=1$ iff $(\exists b \in K)(\exists e \in O d d) b(0)=(n, e)$. Since by Claim 3.4 the branches of $T$ in $N_{K}$ are precisely the elements of $K$, it follows immediately that $\{n \in \omega \mid H(n)=0\}$ is $\Delta_{1}^{1}(T)$ over $N_{K}$. It is clear that this set does not belong to $M_{F}^{G}$ for any finite $F$, since in fact it does not belong to $L_{\omega_{1}^{\text {ck }}}[G]$. In particular then the set does not belong to $N_{K}$. We proved:

Claim 4.1. $\Delta_{1}^{1}$ comprehension fails in $N_{K}$.

In the rest of the section we prove that $N_{K}$ satisfies INDEC. Fix a linear ordering $U=\left(\omega ;<_{U}\right)$ in $N_{K}$. Suppose for contradiction that, in $N_{K}, U$ is scattered, indecomposable, not indecomposable to the left, and not indecomposable to the right.

From these properties of $U$ it follows that there must exist, though not inside $N_{K}$, a unique cut $\langle L, R\rangle$ in $U$, so that $U$ can be embedded, using embeddings in $N_{K}$, to the left of every $\beta \in R$, and to the right of every $\alpha \in L$. We shall look at a name for this cut, and divide into cases depending on whether its interpretation does or does not depend non-trivially on $H$. If it does not, we shall argue for a contradiction by showing that the cut belongs to $N_{K}$. If it does, then working in $N_{K}$ we shall embed the complete binary tree, via $\mathbb{C}$, into $U$, contradicting the fact that $U$ is scattered. For both arguments, we shall work in $M_{F}^{G}$ for $F \subseteq K$ finite and large enough that $U \in M_{F}^{G}$, and reason about embeddings of $U$ in $N_{K}$ by viewing $N_{K}$ as a generic extension of $M_{F}^{G}$. This approach is similar to the one Steel [8] used to prove $\Delta_{1}^{1}$ comprehension in his model.

Let $\bar{A} \subseteq I$ be large enough that $U \in M_{\bar{F}}^{G}$ where $\bar{F}=\left\{f^{G}(i) \mid i \in \bar{A}\right\}$. Let $\bar{M}$ denote $M_{\bar{F}}^{G}$. Let $\dot{U}$ be an $\bar{A}$-nice name for $U$. Let $\langle\bar{p}, \bar{c}\rangle \in G \times H$ force that, in $\dot{N}_{K}, \dot{U}$ is indecomposable, not indecomposable to the left, not indecomposable to the right, and scattered. Extending $\langle\bar{p}, \bar{c}\rangle$ if needed, suppose it forces that $\check{\bar{A}} \subseteq \dot{I}$. In other words suppose that for each $i \in \bar{A}, f_{\bar{p}}(i)(0)$ has the form $(n, e)$, with $n \in \operatorname{dom}(\bar{c})$ and $e$ even iff $\bar{c}(n)=0$.

We work throughout below the condition $\langle\bar{p}, \bar{c}\rangle$.

We also work with $A$-nice names, for $A \supseteq \bar{A}$. ( $A$ is always finite, even when this is not explicitly mentioned.) For every such $A$, there is a natural $A$-nice name $\dot{U}^{\prime}$ which is forced by $1_{\mathbb{P}}$ to be equal to $\dot{U}$. For notational simplicity we identify $\dot{U}^{\prime}$ with $\dot{U}$ in all such situations.

By $(\alpha, \beta)_{U}$ we mean the interval $\left\{\gamma \in \omega \mid \alpha<_{U} \gamma<_{U} \beta\right\}$ of $U$. The interval avoids $\delta$ if $\delta$ is not between $\alpha$ and $\beta$ in the order $<_{U}$. When we say that $\pi$ embeds $U$ to the left of $\beta$ we mean that it embeds $U$ into its restriction to the set $\left\{\gamma \mid \gamma<_{U} \beta\right\}$, and similarly with embedding to the right of $\alpha$.

Claim 4.2. For each $\delta<\omega$, and each condition $\langle p, c\rangle \leq\langle\bar{p}, \bar{c}\rangle$, there exists $\alpha, \beta \in \omega, A \supseteq \bar{A}, A$-nice names $\dot{\sigma}$ and $\dot{\pi}$, and a condition $\langle q, d\rangle \leq\langle p, c\rangle$, so that $\langle q, d\rangle$ forces:

1. $\check{\alpha}<_{\dot{U}} \check{\beta}$ and the interval $(\check{\alpha}, \check{\beta})_{\dot{U}}$ avoids $\check{\delta}$. 
2. $\dot{\sigma}$ embeds $\dot{U}$ to the right of $\check{\alpha}$, and $\dot{\pi}$ embeds $\dot{U}$ to the left of $\check{\beta}$.

3. $\check{A} \subseteq \dot{I}$.

Proof. Fix $\delta$ and fix $\langle p, c\rangle$. Suppose for simplicity that $\langle p, c\rangle \in G \times H$. If not we simply work during this proof with a different generic, $G^{\prime} \times H^{\prime}$, which contains $\langle p, c\rangle$. As $\langle p, c\rangle \leq\langle\bar{p}, \bar{c}\rangle$, the properties of $U$ and $N_{K}$ that we use during the proof hold also for the objects given by the revised generic.

Since $U$ is indecomposable in $N_{K}$, there is in $N_{K}$ an embedding of $U$ either to the left of $\delta$ or to the right. Suppose for definitiveness that it is to the right, and let $\sigma$ be the embedding. Let $\alpha=\delta$. Since $U$ is not indecomposable to the right there is $\beta>_{U} \alpha$ so that $U$ does not embed to the right of $\beta$, in $N_{K}$. Again since $U$ is indecomposable in $N_{K}$, we may fix $\pi \in N_{K}$ which is an embedding of $U$ to the left of $\beta$.

Let $F \supseteq \bar{F}$ be a finite subset of $K$ so that $\pi, \sigma \in M_{F}^{G}$. Let $A \supseteq \bar{A}$ be such that $F=\left\{f^{G}(i) \mid i \in A\right\}$. Let $\dot{\sigma}$ and $\dot{\pi}$ be $A$-nice names for $\sigma$ and $\pi$.

We obtained the $G \times H$-realizations of the conditions in the claim. Finally, fix $\langle q, d\rangle \in G \times H$, stronger than $\langle p, c\rangle$, forcing these conditions to hold.

The first two conditions in Claim 4.2 involve only the forcing $\mathbb{P}$, and only ranked statement in the forcing language. The third condition has very low complexity. It is equivalent to the statement that $f_{q}(i)(0)=(n, e)$ with $n \in$ $\operatorname{dom}(d)$ and $e$ even iff $d(n)=0$, for each $i \in A$. Thus the claim asserts conditions which are $\Delta_{1}$ over $L_{\omega_{1}^{\text {ck }}}$ in their parameters. Using admissability it follows that:

ClAIM 4.3. There is $\theta<\omega_{1}^{\text {ck }}$ so that for each $\delta<\omega$, and each condition $\langle p, c\rangle \leq\langle\bar{p}, \bar{c}\rangle$, one can find in $L_{\theta}$ objects satisfying the conditions of the previous claim.

We work with a fixed $\theta$ witnessing this claim, for the rest of the section. Note that if $\dot{\pi}$ and $\dot{\sigma}$ are $A$-nice names which belong to $L_{\theta}$, then it follows in particular that their orders are below $\theta$. We pick $\theta$ to be closed under ordinal multiplication, and larger than the order of $\dot{U}$. Then it follows that the ranks of the statement

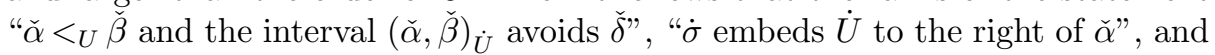
" $\dot{\pi}$ embeds $\dot{U}$ to the left of $\breve{\beta}$ " are all smaller than $\theta$.

Claim 4.4. For each $\delta \in \omega$ and each condition $c \leq \bar{c}$ in $\mathbb{C}$, there exists $\alpha, \beta \in$ $\omega, A \supseteq \bar{A}, A$-nice names $\dot{\sigma}, \dot{\pi}$, and a condition $\langle q, d\rangle$, all in $L_{\theta}$, so that $\langle q, d\rangle$ forces the conditions in Claim 4.2, and in addition to that, $d \leq c$ and $q \in G$.

Proof. Fix $\delta$ and $c$. Modifying $H$ if needed, we may assume for simplicity that $c \in H$.

Let $D$ be the set of $\langle q, d\rangle \in \mathbb{P}$ for which there exists $\alpha, \beta \in \omega, A \subseteq \bar{A}$, and $A$-nice names $\dot{\sigma}, \dot{\pi} \in L_{\theta}$, so that $\langle q, d\rangle$ forces conditions (1)-(3) in Claim 4.2. Because of the restriction to $L_{\theta}$, the set belongs to $L_{\omega_{1}^{c k}}$. By Claim 4.3, the set is dense in $\mathbb{P} \times \mathbb{C}$ below $\langle\bar{p}, \bar{c}\rangle$.

We may thus fix a condition $\langle q, d\rangle \in D \cap(G \times H)$. Then $q \in G$, extending $d$ if needed we may assume that $d \leq c$, and since $\langle q, d\rangle \in D$ we can find the required $\alpha, \beta, A, \dot{\sigma}$, and $\dot{\pi}$.

We will use the claim later, working in the model $\bar{M}=M_{\bar{F}}^{G}$. The claim refers to $G$, which this model cannot identify because it is missing the branches other 
than those in $\bar{F}$, and missing the rank function $h^{G}$. But it has the restriction of the function to nodes with ranks $<\theta$, and this, together with the branches in $\bar{F}$, will be enough through a use of Lemma 3.3 .

Let us be more precise on the approximation to $G$ resulting from these restrictions. Define $\bar{G}$ to be the set of conditions $p \in \mathbb{P}$ extending $\bar{p}$ and so that:

- $T_{p} \subseteq T=T^{G}$.

- $f_{p}(i) \subseteq f^{G}(i)$ for each $i \in \bar{A}$.

- If $h^{G}(t)<\theta$, then $h_{p}(t)=h^{G}(t)$. If $h^{G}(t) \geq \theta$ then $h_{p}(t) \geq \theta$.

In the last condition, as usual, we adopt the convention that $h_{p}(t)=\infty>\theta$ for $t \in \operatorname{Dc}\left(f_{p}\right)$, and similarly with $G$. The set $\bar{G}$ belongs to $\bar{M}=M_{\bar{F}}^{G}$, since the restriction of $h^{G}$ to nodes $t$ so that $h^{G}(t)<\theta$ can be computed from the tree $T$. $\bar{G}$ is not a filter, but it is close enough to $G$ for our purposes. To see this, we will use Lemma 3.3, and the symmetry in the following remark.

Remark 4.5. Suppose that $\alpha, \beta, A, \dot{\sigma}, \dot{\pi}$, and $\langle q, d\rangle$ satisfy the conditions in Claim 4.2. Let $\tau: \omega \rightarrow \omega$ be a bijection, with $\tau\left\lceil\bar{A}=i d\right.$. Let $A^{*}=\tau^{\prime \prime} A$, let $q^{*}=\left\langle T_{q}, f^{*}, h_{q}\right\rangle$ where $f^{*}(\tau(i))=f_{q}(i)$, and let $\dot{\sigma}^{*}$ and $\dot{\pi}^{*}$ be obtained from $\dot{\sigma}$ and $\dot{\pi}$ by replacing references to $\dot{f}(i)$ with references to $\dot{f}(\tau(i))$. Then the conditions in the claim continue to hold for $\alpha, \beta, A^{*}, \dot{\sigma}^{*}, \dot{\pi}^{*}$, and $\left\langle q^{*}, d\right\rangle$.

Claim 4.6. Let $\alpha_{j}, \beta_{j}, A_{j}, \dot{\sigma}_{j}, \dot{\pi}_{j}$, and $\left\langle q_{j}, d_{j}\right\rangle$ belong to $L_{\theta}$ and satisfy the conditions in Claim 4.2 for each $j=0, \ldots, k-1$, with $q_{j} \in \bar{G}$. Suppose that $d_{0}, \ldots, d_{k-1}$ have a common extension in $\mathbb{C}$, and that they are all $\leq \bar{c}$. Then $\alpha_{j}<_{U} \beta_{j}$ for each $j$, and the intervals $\left(\alpha_{j}, \beta_{j}\right)_{U}$ have a non-empty intersection.

Proof. It is enough to prove the claim for $k=2$. The case $k=2$ can then be applied to $j_{0}$ and $j_{1}$ such that $\alpha_{j_{0}}=\max \left(\alpha_{0}, \ldots, \alpha_{k-1}\right)$ and $\beta_{j_{1}}=$ $\min \left(\beta_{0}, \ldots, \beta_{k-1}\right)$, with the maximum and minimum taken using $U$, to yield the general case.

So suppose $k=2$. Our first task is to find an approximation to a common extension of $q_{0}$ and $q_{1}$.

Using Remark 4.5, we may assume that $\operatorname{dom}\left(f_{q_{0}}\right)-\bar{A}$ and $\operatorname{dom}\left(f_{q_{1}}\right)-\bar{A}$ are disjoint. Since both $q_{0}$ and $q_{1}$ belong to $\bar{G}$, both $f_{q_{0}}(i)$ and $f_{q_{1}}(i)$ are initial segments of $f^{G}(i)$ for $i \in \bar{A}$. Extending the conditions we may assume that $f_{q_{0}}(i)=f_{q_{1}}(i)$ for such $i$.

If $t \in T_{q_{o}} \cap T_{q_{1}}$ and one of $h_{q_{0}}(t), h_{q_{1}}(t)$ is defined and $<\theta$, then both are defined, and take the same value. (Both take the value $h^{G}(t)$ on such $t$.) Making adjustments to values of $h_{q_{0}}$ and $h_{q_{1}}$ which are $\geq \theta$, we may assume that $h_{q_{0}}(t)=$ $h_{q_{1}}(t)$ whenever both are defined (not $\infty$ ). Since the modifications only involve values $\geq \theta$, they do not affect the fact that $q_{j} \in \bar{G}$. They also do not affect the fact that the conditions in Claim 4.2 hold, because the forcing statements in the first two conditions have rank $<\theta$ (we are using Lemma 3.3 here), and the statement in the third condition only involve $f_{q_{j}}$ and $d_{j}$, not $h_{q_{j}}$. We can make the modifications in such a way that no changes are made to values on which the original conditions were already in agreement, and so maintain also the fact that $q_{j}$ extends $\bar{p}$ for each $j$.

Let $T^{*}=T_{q_{0}} \cup T_{q_{1}}$. Let $f^{*}=f_{q_{0}} \cup f_{q_{1}}$. Let $\hat{h}=h_{q_{0}} \cup h_{q_{1}}$. The last two unions are possible by the observations in the previous paragraphs. 
$\hat{h}$ is defined on $t \in\left(T^{*}-\operatorname{Dc}\left(f_{q_{0}}\right)\right) \cup\left(T^{*}-\operatorname{Dc}\left(f_{q_{1}}\right)\right)=T^{*}-\left(\operatorname{Dc}\left(f_{q_{0}}\right) \cap \operatorname{Dc}\left(f_{q_{1}}\right)\right)$. Let $h_{j}^{*}=\hat{h} \uparrow T^{*}-\operatorname{Dc}\left(f_{q_{j}}\right)$, and let $h^{*}=\hat{h} \uparrow T^{*}-\left(\operatorname{Dc}\left(f_{q_{0}}\right) \cup \operatorname{Dc}\left(f_{q_{1}}\right)\right)$. Then:

(i) $\left\langle T^{*}, f_{q_{j}}, h_{j}^{*}\right\rangle$ extends $q_{j}$.

(ii) $\left\langle T^{*}, f_{q_{j}}, h_{j}^{*}\right\rangle$ is a $\theta$-absolute $\operatorname{dom}\left(f_{q_{j}}\right)$-reduct of $\left\langle T^{*}, f^{*}, h^{*}\right\rangle$.

$q^{*}=\left\langle T^{*}, f^{*}, h^{*}\right\rangle$ is our approximation to a common extension of $q_{0}$ and $q_{1}$. It follows from (i) that $\left\langle T^{*}, f_{q_{j}}, h_{j}^{*}\right\rangle$ forces that " $\dot{\sigma}_{j}$ embeds $\dot{U}$ to the right of $\check{\alpha}_{j}$, and $\dot{\pi}_{j}$ embeds $\dot{U}$ to the left of $\breve{\beta}_{j}$ ", for each $j \in\{0,1\}$. It follows from (ii) using Lemma 3.3 that forces $q^{*}$ the statement for both $j$.

Both $q_{0}$ and $q_{1}$ extend $\bar{p}$, and it follows from this and the definitions above that $q^{*}$ extends $\bar{p}$. By assumption $d_{0}$ and $d_{1}$ have a common extension in $\mathbb{C}$, and both are $\leq \bar{c}$. Let $d^{*}$ be a common extension of the two conditions, with $d^{*} \leq \bar{c}$.

Consider the condition $\left\langle q^{*}, d^{*}\right\rangle$. Since it is stronger than $\langle\bar{p}, \bar{c}\rangle$, it forces that $\dot{U}$ is scattered in $\dot{N}_{K}$. Since $f^{*}$ extends $f_{q_{j}}$ and $d^{*}$ extends $d_{j},\left\langle q^{*}, d^{*}\right\rangle$ forces that $\check{A}_{j} \subseteq \dot{I}$. (We are using condition (3) of Claim 4.2 here.) Hence, since $\dot{\sigma}_{j}, \dot{\pi}_{j}$ are $A_{j}$-nice, it forces that $\dot{\sigma}_{j}, \dot{\pi}_{j} \in \dot{N}_{K}$.

We claim that $\left\langle q^{*}, d^{*}\right\rangle$ forces that $\check{\alpha}_{j}<_{\dot{U}} \check{\beta}_{j}$ for each $j$, and the intervals $\left(\check{\alpha}_{j}, \breve{\beta}_{j}\right)_{\dot{U}}$ have a non-empty intersection.

Suppose not. Then there is $\left\langle q^{* *}, d^{* *}\right\rangle \leq\left\langle q^{*}, d^{*}\right\rangle$, and $j_{0}, j_{1} \in\{0,1\}$, so that $\left\langle q^{* *}, d^{* *}\right\rangle$ forces $\check{\beta}_{j_{0}} \leq_{\dot{U}} \check{\alpha}_{j_{1}}$. It also forces that $\dot{\pi}_{j_{0}}$ and $\dot{\sigma}_{j_{1}}$ belong to $\dot{N}_{K}$, and embed $\dot{U}$ to the left of $\check{\beta}_{j_{0}}$ and to the right of $\check{\alpha}_{j_{1}}$ respectively. Thus, it forces that in $\dot{N}_{K}$ there are embeddings of $\dot{U}$ to both the left and the right of the interval $\left[\check{\beta}_{j_{0}}, \check{\alpha}_{j_{1}}\right]_{\dot{U}}$. But from such embeddings one can construct an embedding of the rationals into $\dot{U}$, see for example the construction at the end of the proof of Claim 2.9. Thus $\left\langle q^{* *}, d^{* *}\right\rangle$ forces that $\dot{U}$ is not scattered in $\dot{N}_{K}$, and this is a contradiction.

Let $\varphi$ be the forcing statement that $\check{\alpha}_{j}<_{\dot{U}} \check{\beta}_{j}$ for each $j$, and the intervals $\left(\check{\alpha}_{j}, \breve{\beta}_{j}\right)_{\dot{U}}$ have a non-empty intersection. We have shown so far that $\left\langle q^{*}, d^{*}\right\rangle$ forces this statement. Since the statement only involves the poset $\mathbb{P}$, it is forced by $q^{*}$.

$q^{*}$ by construction belongs to $\bar{G}$, but this is not enough for the claim. We have to show that $\alpha_{j}<_{U} \beta_{j}$ and that the intervals $\left(\alpha_{j}, \beta_{j}\right)_{U}$ have a non-empty intersection, and for this we must show that $\varphi$ is forced by a condition in $G$.

The name $\dot{U}$ is $\bar{A}$-nice and belongs to $L_{\theta}$. Since all variables of $\varphi$ range over $\omega$, and all parameters other than $\dot{U}$ are elements of $\omega, \varphi$ is $\bar{A}$-nice and ranked. Since the order of $\dot{U}$ is below $\theta$, and $\theta$ is closed under ordinal multiplication, the rank of $\varphi$ is below $\theta$.

Using the fact that $q^{*} \in \bar{G}$ we can find a $\theta$-absolute $\bar{A}$-reduct $r$ of $q^{*}$ which belongs to $G$. The adjustments leading from $q^{*}$ to $r$ are similar to ones made above, so let us just comment that $T_{r}=T_{q^{*}}, h_{r}=h^{G}\left\lceil T_{r}\right.$ agrees with $h_{q^{*}}$ on nodes in $T_{r}$ which get tags $<\theta, f_{r} \uparrow \bar{A}=f_{q^{*}}\left\lceil\bar{A}\right.$, and outside $\bar{A}, f_{r}$ is defined on a finite domain in such a way that $f_{r}(i) \subseteq f^{G}(i)$ and $\operatorname{Dc}\left(f_{r}\right)=\operatorname{Dc}\left(f^{G}\right) \cap T_{r}$.

Then by Lemma 3.3, $r$ forces $\varphi$.

Recall that our plan is to identify a certain cut $\langle L, R\rangle$ in $U$, and divide into cases depending on whether or not the location of this cut depends non-trivially on $H$. The proof of the next claim handles the case that $\langle L, R\rangle$ does not depend 
on $H$, deriving a contradiction from the fact that $U$ is indecomposable in $N_{K}$. Later we shall use non-trivial dependence of the cut on $H$ to embed the rationals into $U$.

Call intervals $\left(\alpha_{0}, \beta_{0}\right)_{U}$ and $\left(\alpha_{1}, \beta_{1}\right)_{U}$ separated if there is a point of $U$ between them (equivalently, $\beta_{0} \leq_{U} \alpha_{1}$ or $\beta_{1} \leq_{U} \alpha_{0}$ ). This implies in particular that they have empty intersection.

Claim 4.7. For every $c \leq \bar{c}$, there are $\alpha_{j}, \beta_{j}, A_{j}, \dot{\sigma}_{j}, \dot{\pi}_{j}$, and $\left\langle q_{j}, d_{j}\right\rangle$ in $L_{\theta}$, for $j \in\{0,1\}$, which satisfy the conditions in Claim 4.2, with $q_{j} \in \bar{G}$ and $d_{j} \leq c$, and so that the intervals $\left(\alpha_{0}, \beta_{0}\right)_{U}$ and $\left(\alpha_{1}, \beta_{1}\right)_{U}$ are separated. (By the previous claim, $d_{0}$ and $d_{1}$ must be incompatible.)

Proof. Suppose not, and let $c$ witness this. Modifying the generic $H$ if necessary, we may assume that $c \in H$.

Let $Z$ be the set of intervals $(\alpha, \beta)_{U}$ for which there exists $A, \dot{\sigma}, \dot{\pi}$, and $\langle q, d\rangle$, all in $L_{\theta}$, with $q \in \bar{G}$ and $d \leq c$, so that the conditions in Claim 4.2 hold.

Note that $Z$ belongs to $\bar{M}$. Working in $\bar{M}$, let $L=\left\{\gamma \mid\left(\exists(\alpha, \beta)_{U} \in Z\right) \gamma \leq_{U} \alpha\right\}$, and let $R=\left\{\gamma \mid\left(\exists(\alpha, \beta)_{U} \in Z\right) \gamma \geq_{U} \beta\right\}$. It is clear that $L$ is closed leftward in $U$, and $R$ is closed rightward. By our assumption for contradiction, no two intervals in $Z$ are separated. It follows that $L$ and $R$ are disjoint, for otherwise there is $\gamma$ with an interval in $Z$ to its right, and another interval in $Z$ to its left, so that these intervals are separated. Finally, $L \cup R$ is the entire line $U$, by Claim 4.4: Given $\delta \in \omega$, there is by the claim an interval in $Z$ which avoids $\delta$. So $\delta$ is either to the left of the interval, in which case $\delta \in L$, or to the right of the interval, in which case $\delta \in R$.

We constructed, inside $\bar{M}$, a cut $\langle L, R\rangle$ in $U$. Since $U$ is indecomposable in $N_{K} \supseteq \bar{M}$, it must be that $U$ embeds either into $L$ or into $R$. Suppose for definitiveness that $U$ embeds into $L$. Taking the square of this embedding we can find $\delta \in L$ so that, in $N_{K}, U$ embeds to the left of $\delta$.

By Claim 4.4, for each $c \leq \bar{c}$ there exists $\alpha, \beta \in \omega, A \supseteq \bar{A}, A$-nice names $\dot{\sigma}$, $\dot{\pi}$, and a condition $\langle q, d\rangle$, all in $L_{\theta}$, so that $\langle q, d\rangle$ forces the conditions in Claim 4.2 , and in addition to that, $d \leq c$ and $q \in G$. Since we can get $d \leq c$ for an arbitrary $c \leq \bar{c}$, the set of $d$ which can occur in such tuples is dense below $\bar{c}$. By the genericity of $H$, we can find a tuple as above, with $d \in H$.

As $q \in G$ and $d \in H$, the conditions in Claim 4.2 imply that $\alpha<_{U} \beta$, the interval $(\alpha, \beta)_{U}$ avoids $\delta, \sigma=\dot{\sigma}[G]$ embeds $U$ to the right of $\alpha$, and $\sigma \in N_{K}$.

The interval $(\alpha, \beta)_{U}$ belongs to $Z$ by definition of $Z$, and since $\delta \notin R$ it must be that $\delta<_{U} \beta$. Since the interval avoids $\delta$, we must have $\delta \leq_{U} \alpha$. So $\sigma$ embeds $U$ to the right of $\delta$.

We found, in $N_{K}$, two embeddings of $U$, to the left and to the right of $\delta$. From these embeddings one can construct an embedding of the rationals into $U$, contradicting the fact that $U$ is scattered in $N_{K}$.

We now have the tools needed for the final stage of the proof. We shall embed the rationals, via $\mathbb{C}$, into $U$. We work in $\bar{M}$ throughout.

For each $c \leq \bar{c}$ in $\mathbb{C}$, let $Z_{c}$ be the set of intervals $(\alpha, \beta)_{U}$ for which there exists $A, \dot{\sigma}, \dot{\pi}$, and $\langle q, d\rangle$, all in $L_{\theta}$, with $q \in \bar{G}$ and $d \leq c$, so that the conditions in Claim 4.2 hold. For each interval $(\alpha, \beta)_{U}$ in $Z_{c}$, let $d_{c}(\alpha, \beta)$ be a condition 
in $\mathbb{C}$ so that a witness for the membership of $(\alpha, \beta)_{U}$ in $Z_{c}$ can be picked with $d=d_{c}(\alpha, \beta)$.

Since $\theta<\omega_{1}^{\mathrm{ck}}$, and $\bar{G}$ belongs to $\bar{M}$, each $Z_{c}$ is an element of $\bar{M}$, and indeed the function $c \mapsto Z_{c}$ belongs to $\bar{M}$. Similarly the function $c, \alpha, \beta \mapsto d_{c}(\alpha, \beta)$ belongs to $\bar{M}$.

Let $W$ be the complete binary tree with order determined by $s \frown\langle 0\rangle\urcorner t<_{W} s$ and $s \frown\langle 1\rangle-t>_{W} s$. Working recursively on the length of $s \in W$, define maps $s \mapsto c_{s}$, and $s \mapsto\left(\alpha_{s}, \beta_{s}\right)_{U}$ for $s \neq \emptyset$, where $c_{s} \leq \bar{c}$ in $\mathbb{C}$ and $\alpha_{s}<_{U} \beta_{s}$, as follows: Set $c_{\emptyset}=\bar{c}$. Once $c_{s}$ is known, let $\left(\alpha_{0}, \beta_{0}\right)_{U}$ and $\left(\alpha_{1}, \beta_{1}\right)_{U}$ be separated intervals in $Z_{c}$. Separated intervals in $Z_{c}$ can be found by Claim 4.7. Arrange them so that $\left(\alpha_{0}, \beta_{0}\right)_{U}$ is to the left of $\left(\alpha_{1}, \beta_{1}\right)_{U}$. Set $\left(\alpha_{s} \frown\langle j\rangle, \beta_{s} \frown\langle j\rangle\right)_{U}=\left(\alpha_{j}, \beta_{j}\right)_{U}$ for $j=0,1$, and set $c_{s} \frown\langle j\rangle=d_{c_{s}}\left(\alpha_{j}, \beta_{j}\right)$.

The objects constructed then have the following properties:

1. $c_{s} \frown\langle j\rangle$ extends $c_{s}$, by definition of the map $d_{c}$.

2. The intervals $\left(\alpha_{s \uparrow i}, \beta_{s \uparrow i}\right)_{U}, 0<i \leq \operatorname{lh}(s)$, have a non-empty intersection, by Claim 4.6, because the conditions $c_{s \uparrow i}$ are compatible. (Indeed these conditions form a chain.)

For each $s \neq \emptyset$, let $O_{s}=\bigcap_{0<i \leq \operatorname{lh}(s)}\left(\alpha_{s \uparrow i}, \beta_{s \uparrow i}\right)_{U}$. From the fact that $O_{s}<\langle 0\rangle$ and $O_{s} \prec\langle 1\rangle$ are both non-empty, and that the intervals $\left(\alpha_{s}<\langle 0\rangle, \beta_{s} \prec\langle 0\rangle\right)$ and $\left(\alpha_{s} \frown\langle 1\rangle, \beta_{s} \frown\langle 1\rangle\right)$ are separated with the former to the left of the latter, it follows that:

3. $\beta_{s} \frown\langle 0\rangle \leq_{U} \alpha_{s} \prec\langle 1\rangle$ and both belong to $O_{s}$.

For each $s \in W$ pick $\delta_{s}$ so that $\beta_{s} \frown\langle 0\rangle \leq_{U} \delta_{s} \leq_{U} \alpha_{s} \frown\langle 1\rangle$. (For example, take $\left.\delta_{s}=\beta_{s} \frown\langle 0\rangle.\right)$ Then:

4. $\delta_{s}$ belongs to $O_{s}, O_{s} \prec\langle 0\rangle \subseteq O_{s}$ is to the left of $\delta_{s}$, and $O_{s} \frown\langle 1\rangle \subseteq O_{s}$ is to the right of $\delta_{s}$.

It is clear from this that the map $s \mapsto \delta_{s}$ embeds $W$ into $U$.

We constructed, inside $\bar{M} \subseteq N_{K}$, an embedding of $W$ into $U$. Since the rationals can be embedded into $W$, it follows that $U$ is not scattered in $N_{K}$. This is a contradiction, obtained from our initial assumption that, in $N_{K}, U$ is a counterexample to INDEC.

Thus, INDEC holds in $N_{K}$. Since $\Delta_{1}^{1}$ comprehension fails in $N_{K}$, we conclude that INDEC does not imply $\Delta_{1}^{1}$ comprehension.

\section{REFERENCES}

[1] Roland Fraïssé, Theory of relations, revised ed., Studies in Logic and the Foundations of Mathematics, vol. 145, North-Holland Publishing Co., Amsterdam, 2000, With an appendix by Norbert Sauer.

[2] Pierre Jullien, Contribution à l'étude des types d'ordre dispersés, Ph.D. thesis, Marseille, 1969.

[3] Antonio Montalbán, On the $\Pi_{1}^{1}$ separation principle, Mathematical Logic Quarterly, to appear.

[4] - Indecomposable linear orderings and hyperarithmetic analysis, J. Math. Log., vol. 6 (2006), no. 1 , pp. 89-120.

[5] Joseph G. Rosenstein, Linear orderings, Pure and Applied Mathematics, vol. 98, Academic Press Inc. [Harcourt Brace Jovanovich Publishers], New York, 1982. 
[6] Stephen G. Simpson, Subsystems of second order arithmetic, Perspectives in Mathematical Logic, Springer-Verlag, Berlin, 1999.

[7] John R. Steel, Determinateness and subsystems of analysis, Ph.D. thesis, University of California, Berkeley, 1977.

[8] — Forcing with tagged trees, Ann. Math. Logic, vol. 15 (1978), no. 1, pp. 55-74.

[9] Robert VAn Wesep, Subsystems of second-order arithmetic, and descriptive set theory under the axiom of determinateness, Ph.D. thesis, University of California, Berkeley, 1977.

DEPARTMENT OF MATHEMATICS

UNIVERSITY OF CALIFORNIA LOS ANGELES

LOS ANGELES, CA 90095-1555

E-mail: ineeman@math.ucla.edu 